\title{
Les antiquités de Russie méridionale au Louvre et la collection Messaksoudy
}

Joanna Martin

\section{(2) OpenEdition \\ 1 Journals}

Édition électronique

URL : https://journals.openedition.org/edl/1670

DOI : $10.4000 /$ edl. 1670

ISSN : 2296-5084

Éditeur

Université de Lausanne

\section{Édition imprimée}

Date de publication : 15 mai 2019

Pagination : 23-62

ISBN : 978-2-940331-70-3

ISSN : 0014-2026

\section{Référence électronique}

Joanna Martin, "Les antiquités de Russie méridionale au Louvre et la collection Messaksoudy », Études de lettres [En ligne], 309 | 2019, mis en ligne le 15 mai 2021, consulté le 17 mai 2021. URL http://journals.openedition.org/edl/1670 ; DOI : https://doi.org/10.4000/edl.1670 


\section{LES ANTIQUITÉS DE RUSSIE MÉRIDIONALE AU LOUVRE ET LA COLLECTION MESSAKSOUDY*}

L'histoire des collections provenant de Russie méridionale dans les musées occidentaux demeure un sujet peu traité. En France, les musées du Louvre et d'Archéologie nationale à Saint-Germain-en-Laye intègrent un mobilier archéologique provenant de ces régions essentiellement à la faveur des «dévolutions» de la guerre de Crimée (1853-1856), ainsi que de l'achat d'une riche collection privée d'antiques, celle de Pierre Messaksoudy. Nous revenons ici sur les circonstances ayant présidé à l'entrée de ce patrimoine dans les musées français et aux péripéties qui en accompagnent l'acquisition.

Lorsque le Muséum central des Arts, futur musée du Louvre, ouvre ses portes au public parisien en août 1793 , les antiques y tiennent une place majeure. Le manifeste winckelmannien L'histoire de l'art chez les Anciens, paru en 1764, a théorisé et placé sur un piédestal l'Antiquité grecque considérée dans une vision classicisante, épurée tant dans sa construction formelle que dans la blancheur du marbre, et portée en tant que telle

* Cet article est un résumé de mon travail de mémoire en Master 1, Les auvres provenant de Crimée dans les collections du département des Antiquités grecques, étrusques et romaines du musée du Louvre, présenté en 2014 à l'École du Louvre sous la direction conjointe de Corinne Jouys Barbelin, actuellement chef du service des Ressources documentaires au Musée d'Archéologie nationale de Saint-Germain-en-Laye (M.A.N.), et de Violaine Jeammet, conservateur en chef au Département des Antiquités grecques, étrusques et romaines du musée du Louvre (DAGER). Il est conservé à la Bibliothèque de l'École du Louvre. Je prolonge désormais cette réflexion dans une recherche doctorale menée dans le cadre du programme Mer Noire du DAGER (dir. Alexandre Baralis), en codirection entre l'École du Louvre et l'Université Paris-Nanterre (dir. Christel Müller). Je souhaite remercier Alexandre Baralis et Christel Müller pour leur relecture de cet article et leurs judicieuses remarques. 
dans les arts par le mouvement néoclassique. Il règne à Paris une Grèce rêvée, celle du "Beau», symétrique et bien proportionné. Le génie grec s'exprime par cette "noble simplicité et grandeur sereine" décrite dans l'art de la Grèce continentale par Winckelmann. Et lorsqu'on célèbre à Paris, quelques années plus tard, les antiques confisqués en Italie et que s'ouvre en 1800 le musée des Antiques, c'est toujours ce même canon qui domine.

L'art des colonies grecques du Pont-Euxin n'aura en revanche pas rencontré les mêmes faveurs auprès des conservateurs du musée. Ce désintérêt interroge et nous conduit à enquêter sur l'existence et la place qu'occupent aujourd'hui les collections provenant des rives de la mer Noire dans l'historiographie muséale. Les musées occidentaux, en effet, se contentent généralement de la simple insertion de ce corpus d'œuvres dans leurs catalogues généraux, comme on le constate à la lecture des ouvrages de Paule Pinelli et Aleksandra Wąsowicz, Véronique ArveillerDulong et Marie-Dominique Nenna ou encore d'André de Ridder ${ }^{1}$. Exceptionnellement une collection particulière fait l'objet d'une étude, comme celle d'Alexandre Berthier de Lagarde au British Museum ${ }^{2}$ ou celle d'Alexandre Merle de Massonneau par Alexander Leskov ${ }^{3}$, mais une réflexion plus globale sur ces fonds, tout comme sur les circonstances et les raisons conduisant à leur entrée dans une collection nationale manque encore.

Pour traiter ce sujet, nous disposons actuellement d'un corpus d'archives important. Les livres d'entrée et les documents conservés au Département des Antiquités grecques, étrusques et romaines du musée du Louvre (DAGER), aux Archives nationales (AN), au Service historique de la Défense (SHD), ainsi que d'autres archives ailleurs en Europe et aux États-Unis constituent des sources à partir desquelles nous pouvons reconstituer les parcours parfois complexes que l'acquisition et la réception de ces antiquités pontiques ont par le passé occasionnés au sein du musée du Louvre. Sans prétendre à l'exhaustivité dans le cadre

I. I. V. Tunkina, Russkaja nauka o klassičeskikh drevnostjakh juga Rossii; P. Pinelli, A. Wąsowicz, Catalogue des bois et stucs grecs et romains provenant de Kertch; J. Frel, "Miscellanea Pontica» I et II; V. Arveiller-Dulong, M.-D. Nenna, Les verres antiques du musée du Louvre; A. de Ridder, Catalogue sommaire des bijoux antiques.

2. J. Andrasi, A. Aibabin, The Berthier-Delagarde Collection of Crimean Jewllery in the British Museum and Related Material.

3. A. Leskov, The Maikop Treasure. 
restreint de cet article, nous porterons notre attention, après quelques éclaircissements utiles à établir la chronologie des acquisitions d'antiquités pontiques pour le compte du Louvre, sur deux moments clés: le premier concerne l'apport issu de la guerre de Crimée (en 1856), le second l'achat (en 1920) d'une collection privée formée à Kertch.

\section{Amplitude chronologique des acquisitions}

Si au XIXe siècle les musées sont encore dans leur jeunesse, "le goût de l'enquête avec ses surprises» est déjà bien présent chez les antiquaires du siècle précédent, comme le note Marc Fumaroli ${ }^{4}$. L'investigation scientifique s'établit et ses modalités opératoires s'affinent. Garant d'un niveau scientifique qui se veut élevé, le musée du Louvre attend avant d'acquérir des antiquités provenant de Russie méridionale. L’analyse du tableau comparatif des acquisitions de cette région démontre l'attitude réservée des conservateurs (fig. 1) ${ }^{5}$.

Le premier objet identifié, une bague en or de la collection Durand, a été acquis par le Louvre en 1825. Cependant rien dans les archives ne permet d'affirmer avec certitude que cet objet provienne de Russie méridionale. Sa provenance est arrêtée sur des considérations stylistiques postérieures à l'achat. Le dernier objet, une applique de vêtement représentant un Scythe, date de 1992. Plus de cent cinquante ans séparent ces deux acquisitions ${ }^{6}$.

On observe deux pics principaux dans les acquisitions - respectivement en 1856 et 1920 -, entre lesquels s'intercale une progression des acquisitions à partir de 1873 , avant un net recul de ces dernières après

4. C'est une allusion au célèbre titre de l'exposition La jeunesse des musées. Les musées en France au XIX $X^{e}$ siècle, qui s'est tenue à Paris, au Musée d'Orsay du 8 février au 7 mai 1994 et qui retraça l'histoire de cette institution, ainsi que de ses fonctions. M. Fumaroli, «Retour à l'Antique», p. 39.

5. Ce tableau a été élaboré par mes soins à l'aide de différents instruments et outils qui ont permis de recenser cette collection. Je ne rentre pas dans les détails matériels de ces outils et me borne à signaler qu'il s'agit des livres d'entrée et catalogues du DAGER, un fichier manuscrit conçu dans les années 1960-1970 en vue de l'informatisation future du département dit «Fichier Suzanne Favier» et des outils informatiques actuels, tels la base documentaire "Jupiter».

6. Pour la bague: ED4803 - INV. 2214/Bj 1277; pour l'applique: MNE 968/Bj 2344. 


\begin{tabular}{|c|c|c|c|c|c|c|c|c|c|c|}
\hline & Marbre & Albâtre & Verre & $\begin{array}{l}\text { Céramiques et } \\
\text { terre cuite }\end{array}$ & Stucs & Bois & Bronzes & Orfèvrerie & Os & $\begin{array}{c}\text { Total } \\
\text { par année }\end{array}$ \\
\hline 1825 & & & & & & & & 1 & & 1 \\
\hline 1856 & 3 & 3 & 24 & 17 & 21 & 62 & 202 & 9 & & 341 \\
\hline 1861 & & & & & & & & 1 & 14 & 15 \\
\hline 1863 & & & & & & & & 1 & & 1 \\
\hline 1873 & & & & & & & 1 & & & 1 \\
\hline 1877 & & & & 9 & & & & & & 9 \\
\hline 1887 & & & & 6 & & & & & & 6 \\
\hline 1889 & & & & 11 & 4 & & 1 & 8 & & 24 \\
\hline 1892 & & & & 1 & & & & & & 1 \\
\hline 1894 & & & & 6 & & & & & & 6 \\
\hline 1895 & & & & & & & 1 & & & 1 \\
\hline 1896 & & & & & & & 19 & & & 19 \\
\hline 1897 & & & & & 15 & & 1 & & & 16 \\
\hline 1900 & & & & & & & & 3 & & 3 \\
\hline 1903 & & & & & & & & 10 & & 10 \\
\hline 1904 & & & & 1 & & & & & & 1 \\
\hline 1919 & & & & 1 & & & & & & 1 \\
\hline 1920 & & & 74 & 65 & 35 & & 25 & 162 & 4 & 365 \\
\hline 1923 & & & & 1 & & & & & & 1 \\
\hline 1925 & & & & & & & 2 & & & 2 \\
\hline 1937 & & & & 2 & & & & & & 2 \\
\hline 1949 & & & & & & & & 1 & & 1 \\
\hline 1962 & & & & & & & & 1 & & 1 \\
\hline 1967 & & & & & & & & & 1 & 1 \\
\hline 1992 & & & & & & & & 1 & & 1 \\
\hline $\begin{array}{c}\text { Total } \\
\text { par matériau }\end{array}$ & 3 & 3 & 98 & 120 & 75 & 62 & 252 & 198 & 19 & 830 \\
\hline
\end{tabular}

Fig. 1 - Tableau récapitulatif de la collection provenant de Russie méridionale conservée au Département des Antiquités grecques, étrusques et romaines du musée du Louvre. 
1903. Ces dates ne sont pas anodines dans l'histoire des collections du Louvre. Les deux pics sont sans commune mesure par rapport aux autres acquisitions et correspondent à deux apports différents: le premier est décrit dans les livres d'entrée sous l'appellation «Dévolution de l'Armée de Crimée»; le second est un achat d'une collection privée constituée pour l'essentiel à Kertch et à Taman. La progression des achats après 1873 correspond en quelque sorte à «l'admission» des antiquités russes dans les cercles des savants français, leurs descriptions et leurs discussions dans les revues spécialisées, conjuguées à l'intérêt porté à l'archéologie des périodes préhistoriques du Caucase et de Russie méridionale, largement relayée dans la presse spécialisée.

Le recul des acquisitions à partir de l'année 1903 s'explique de même aisément, mais la compréhension des facteurs qui l'expliquent impose un bref détour par une affaire encore sensible aujourd'hui, à savoir l'achat de la tiare de Saïtapharnès. Cette acquisition donna lieu à un procès en authenticité, lequel se termina précisément au mois de juin $1903^{7}$. Le verdict fut sans appel: la tiare s'avéra être un faux.

Suite à cette affaire, les antiquités de Russie méridionale susciteront la méfiance jusque dans les années 1920, à un moment où l'achat d'une collection entièrement constituée en Crimée relance cet intérêt, tout en marquant son chant du cygne. Les changements politiques majeurs intervenus en Russie à la faveur de la Révolution d'octobre 1917 finiront par assécher les canaux d'approvisionnement et il ne restera que le circuit des collectionneurs ayant acquis des objets avant cette date pour satisfaire la demande des musées ${ }^{8}$.

7. Rapport publié par le journal Le Temps en date du 11 juin 1903. Voir aussi V. Schiltz, «Du bonnet d'Ulysse à la tiare de Saïtapharnès».

8. On doit également constater une certaine désaffection des marchands d'art pour la région. Ainsi la célèbre maison Sambon et surtout le petit-fils du fondateur, Arthur, qui exerce à Paris et ne souhaite pas s'occuper de la vente des objets provenant de Russie: «Quand je vous ai dit que la collection de camées pourrait m’intéresser, je n'ai pas pensé à ce détail pourtant bien important, qu'elle provient de Russie. Je ne désire pas acheter des collections provenant de ce pays [...]». Document conservé aux AN, Fonds Sambon 613 AP/2, dossiers et registres de correspondance, Dossier AS 9, p. 176, courrier à M. Mons en date du 16 septembre 1920. 


\section{L'apport de 1856: la guerre de Crimée}

\subsection{Le contexte historique}

Dans les livres d'entrée du DAGER, les objets répertoriés comme acquis en 1856 portent le terme "Dévolution de l'Armée de Crimée». Au Louvre, pareille annotation signifie une voie d'acquisition hors achat, don/legs et fouille réglementée ${ }^{9}$. La date d'acquisition correspond en réalité à l'issue de la guerre de Crimée (1854-1856) lors de laquelle la France est présente aux côtés des Anglais, des Piémontais et des Turcs. Sans rentrer dans les détails des opérations militaires, rappelons seulement le siège de Sébastopol qui débute en octobre 1854 et une expédition à Kertch, décidée le 22 mai 1855 et qui se terminera le 15 juin 1855. C'est précisément cette expédition qui nous intéresse, puisque c'est à son terme que les objets archéologiques se retrouvent dans la collection nationale française ${ }^{10}$. L'expédition militaire se dirige vers Kertch pour des raisons strictement stratégiques; elle doit couper la ligne d'approvisionnement des troupes russes qui franchissait le détroit de Kertch. Elle est composée de troupes françaises, anglaises et turques. Le «Rapport de l'expédition de Kertch et d'Yenikalé» de la première division du premier corps indique que le 25 mai 1855 la division française était en tête et qu'elle

[...] fut accueillie avec des témoignages de sympathie par les habitants cependant surpris par notre arrivée. Le Général Wrangel qui était investi du commandement de la presqu'île avait quitté Kertch et s'était retiré avec ses troupes sans prévenir les habitants ${ }^{11}$.

9. En réalité l'Armée de Crimée n'existe pas sur le plan militaire; les archives du Service historique de la Défense mentionnent en effet l'Armée d'Orient. À ce sujet, voir Inventaire SHD Vincennes. Archives historiques, série G Second empire, sous-série G1 Armée d'Orient et expédition de la Baltique, 1854-1856.

Io. D'autres objets se trouvent au British Museum comme le précise A. Villing dans un courrier qu'elle m'a adressé le 19 février 2014: "The BM database lists a total of 798 objects from the Crimea. The majority (ca. 450) entered the collection in the mid 19th century (1856/57), in the wake of the Crimean War, via Duncan MacPherson, Robert Westmacott and William Munro [...]».

II. SHD, G1, G 99, Gallipoli et Eupatoria (commandements militaires). - Kertsch et Kinburn (corps expéditionnaires), pièce no 296 Artillerie. Expédition de Kertch et d'Yenikalé. Rapport sur l'expédition de Kertch et d'Ienikalé. 
Cette entrée pacifique est entachée par un incident pénible, selon les termes du général Trochu ${ }^{12}$ : le pillage de la ville et la mise à sac du musée archéologique. Les récits que nous en possédons sont de natures diverses, le plus connu étant celui du docteur anglais Duncan McPherson. Du côté français, il est présent dans les ouvrages d'historiens tels Léon Guérin ou du journaliste Émile de La Bédollière. En revanche, l'histoire dite "officielle» - Eugène Pick de l'Isère ou les dépêches militaires envoyées de Kertch - ne le mentionne pas ${ }^{13}$. Nous rapportons ici les faits selon le récit d'Eugène Perret:

Dans l'après-midi du 25 mai, les équipages de quelques navires marchands descendirent à terre, brisèrent les portes de trois ou quatre maisons qui avaient été barricadées, et les livrèrent au pillage [...].

Sans attenter à la sûreté des personnes, quelques maraudeurs anglais et français, il faut bien l'avouer, prirent part au désordre [...].

Le musée fut dévasté. Ces barbares arrachèrent des tables de marbre scellées dans les murs, brisèrent des statues et des bas-reliefs. «La grande salle, écrivait le lendemain un visiteur anglais, était garnie d'armoires vitrées et de socles pour les statues; les plus petites antiquités étaient placées sur plusieurs rangs d'étagères parallèles. À l'extrémité du bâtiment, en face de la porte, se trouvait une sorte de plate-forme élevée de trente pieds environ au-dessus du sol et occupant toute la largeur de l'édifice, sur laquelle on avait réuni un grand nombre d'urnes cinéraires, tirées sans doute des tumuli, qui sont si nombreux dans les environs. On y monte par un escalier tournant pratiqué dans une des colonnes qui se trouvent à l'extrémité de la salle. On a peine à comprendre comment la furie de quelques hommes a pu faire tant de dégâts et accumuler tant de ruines en si peu de temps» ${ }^{14}$.

I2. Bibliothèque de l'Institut, MS 3723/2 Correspondance Maxime Du Camp. Guerre de Crimée. Notes du Général Trochu sur les opérations militaires et sur les assauts du 18 juin et du 8 septembre 1855, rédigés par Arthur Brunet (1855) et annotés par le général Achille de Susleau de Malroy (1877), p. 49 (136).

13. D. McPherson, Antiquities of Kertch and researches in the Cimmerian Bosphorus, p. 40 ; E. Perret, Les Français en Orient, p. 287; L. Guérin, Histoire de la dernière guerre de Russie (1853-1856), t. 2, p. 235; É. de La Bédollière, Histoire de la guerre d'Orient, p. 35; E. Pick (de l'Isère), Les fastes de la guerre d'Orient. SHD, G1, 58, Cabinet du Général en chef, Correspondance générale du 28 IV 1855 au 16 VII 1856, Lettre nº 20 du 29 mai 1855: «le 25 à midi, après une marche pénible elles [les troupes] se sont établies dans Yêni-kalé».

I4. E. Perret, Les Français en Orient, p. 287-289. 
Un article paru dans le journal Manuel général de l'instruction primaire qui reprend les termes du rapport officiel établi par le vice-amiral Bruat, informe ses lecteurs avec une retenue toute diplomatique qu'«il existait à Kertch un très-beau musée d'antiques; la Mégère est chargée de le déménager, et il va être envoyé en France " ${ }^{15}$. Le général Trochu rapporte le contexte dans lequel ce pillage a pu être commis:

Évidemment avant l'entrée des troupes, les généraux avaient négligé de faire publier les bans d'occupation à la tête des compagnies et de faire occuper solidement la ville par de fortes sauvegardes et par des postes de soutine. Mais c'était un désordre international à trois, ceuxci en attribuant l'initiative à ceux-là et en chargeant dans tous les cas et comme il ne pouvait pas manquer d'arriver la sauvagerie des Turcs. Il fallait passer l'éponge ${ }^{16}$.

On peut s'étonner que les responsables militaires n'aient pas été plus prévoyants et n'aient pas pris les mesures qui s'imposaient lors de l'entrée de l'armée dans la ville. Déjà en 1854, lors du débarquement de l'Armée d'Orient à Gallipoli, sur les rives européennes des Dardanelles, la découverte de vestiges antiques s'était accompagnée d'actes de vandalisme. Ils poussèrent le colonel commandant supérieur de la presqu'île de Gallipoli à donner des ordres stricts quant à la conduite des soldats en cas de trouvaille archéologique et du traitement à leur réserver ${ }^{17}$. Du côté français, l'épisode reste flou: les sources officielles que nous avons réussi à

15. Manuel général de l'instruction primaire, no 24 du 16 juin 1855, p. 278. Ce journal publie le rapport officiel du vice-amiral Bruat, dicté sur le vaisseau Montebello le 26 mai 1855. La Mégère est un aviso à vapeur de première classe.

16. Bibliothèque de l'Institut: Correspondance Maxime Du Camp, MS 3723/2, cf. supra n. 12.

17. SHD, G1, 99, Armée d'Orient, Place de Gallipoli, État-Major de la Place, Ordres généraux de l'armée, pièce no 290 : Ordre no 35, p. 108 du 219 bre [novembre] 1854 concernant les marbres ou pierres portant des inscriptions antiques. Ce traitement envisagé à Gallipoli relève manifestement d'une initiative unique en son genre pour l'armée française, puisque encore au mois de mai 1856 - c'est-à-dire après la conférence de paix tenue à Paris - le chef d'escadron d'État-major Beaudoin écrit du grand quartier général à Sébastopol; il s’inquiète de trouvailles de médailles antiques par les soldats et la perte que cela constitue pour la science. Voir la note de l'intendant Beaudoin concernant les trouvailles des soldats dans les tranchées de Sébastopol, conservée aux Archives des musées nationaux (ci-après AMN), A2, 1870, 19 avril [Copie] (actuellement transférée aux Archives nationales, site Pierrefitte-sur-Seine où elles portent une cotation différente de celle indiquée ici). 
exploiter ne mentionnent ni la façon dont les pièces ont été rassemblées sur place, ni comment s'est effectué leur transport jusqu'en France.

\subsection{L’entrée des objets au Louvre}

L'histoire du fonds criméen du DAGER réapparaît le 16 décembre 1855 dans une lettre du conservateur des Antiques et de la Sculpture moderne du Louvre, Adrien de Longpérier, au directeur général des Musées Impériaux, Émilien de Nieuwerkerke, au sujet des deux sphinx «du plus mauvais style, qui ont été envoyés de Sébastopol ${ }^{18}$ et qui encombrent les salles du Musée assyrien.

Ce fonds est constitué à partir d'objets expédiés par le Ministère de la Guerre au musée du Louvre en 1856. Il ne suscite d'ailleurs au Louvre aucun enthousiasme; Nieuwerkerke écrit au ministre d'État et relaie une opinion émise par la conservation du Louvre qui souligne que «cette collection d'objets recueillis en Crimée est tout à fait dépourvue de valeur intrinsèque et n'a d'autre intérêt que son origine " ${ }^{19}$. La seule chose qui pousse le conservateur à accepter cet envoi est son utilité pour les "antiquaires en leur fournissant quelques indications précises sur l'analogie de la poterie de Kertch avec celle d'Athènes et de la Cyrénaïque» ${ }^{20}$.

Il y a d'abord l'envoi des quatorze caisses qui arrivent au Louvre entre le 21 décembre 1855 et le $1^{\text {er }}$ février 1856 de Marseille et de Toulon - cet envoi s'opérant en deux temps en raison de son poids. Puis le ministre d'État de la Maison de l'Empereur informe le directeur des Musées Impériaux, Nieuwerkerke, en novembre 1856 que deux autres caisses, avec des

[...] objets recueillis sur l'emplacement de Kherson en Crimée, par les soins de M. le Duc de Malakoff sont parvenus à son département et

I8. AMN S 12, 1855, 16 décembre. Lettre d'A. de Longpérier à Monsieur le Directeur Général, en date du 16 décembre 1855. Ces deux sphinx se trouvent aujourd'hui au Jardin des Tuileries. Voir G. Bresc, A. Pingeot, Sculptures des jardins du Louvre, du Carrousel et des Tuileries, t. 2, p. 470-473. Nous remercions ici M. Alain Prévet, responsable des Archives des musées nationaux, pour son aimable communication à ce sujet.

19. AMN, Z4, 1856, 28 février. Lettre du Directeur Général des Musées Impériaux (...) à Monsieur le Ministre d'État et de la Maison de l'Empereur, en date du $1^{\mathrm{er}}$ mars 1856.

20. AMN, Z 4, 1856, 28 février. Lettre d'A. de Longpérier à M. Le Directeur Général, en date du 28 février 1856. 
qu'il tient à ma disposition ces objets qui, en raison de leur antiquité et de leur origine lui paraissent devoir trouver utilement place dans les collections des Musées Impériaux ${ }^{21}$.

Au Ministère de la Guerre, on trouve encore des caisses en 1858 et on prie de les examiner en vue "d'intéresser quelques-uns de nos musées impériaux " ${ }^{22}$. En avril 1859, A. de Longpérier écrit à É. de Nieuwerkerke au sujet des «morceaux antiques que l'intendant militaire de la Garde a rapportés de Crimée et qu'il désire donner au Musée» ${ }^{23}$. A. de Longpérier insiste auprès du directeur des Musées Impériaux pour que ce don soit accepté: «il serait fâcheux que les monuments recueillis par nos soldats ne puissent trouver asile dans un musée public ${ }^{24}$. La publication de ces objets, inscrits sur le livre d'entrée Napoléon III Mobilier de la couronne 1 à 1650, Napoléon III Mobilier de la Couronne 1651 à 3591 et N III Livre d'entrée, suscite des interrogations chez le conservateur: ils seront finalement considérés comme un don du Ministère de la Guerre et c'est en cette qualité qu'ils apparaissent dans le Rapport sur les travaux de remaniement et d'accroissement des Musées Impériaux ${ }^{25}$.

\subsection{Contenu du fonds et nature des objets}

Lorsque l'on examine le contenu de cette collection, on y trouve des objets de nature diverse décrits dans les livres d'entrée consultés sous des dénominations différentes. Ainsi le livre d'entrée Napoléon III mobilier de la Couronne I mentionne 24 numéros qui rassemblent uniquement des bronzes, à savoir essentiellement des miroirs, mais aussi des fragments

2I. AMN, Z 4, 1856, 28 février. Lettre du Directeur Général des Musées Impériaux (...) à Monsieur le Ministre d'État et de la Maison de l'Empereur, en date du $1^{\mathrm{er}}$ mars 1856 et lettre en date du 4 novembre 1856.

22. AMN, A2, 1858, 26 août. Lettre du Ministre Secrétaire d'État de la guerre (...) à Monsieur le Directeur Général, en date du 26 août 1858.

23. Il s'agit ici du don de C. Robert.

24. AMN, A8, 1859, 13 mai. Lettre d'A. de Longpérier à $M$. le Directeur Général, en date du 26 avril 1859. Il s'agit du numéro MNC 1794/Ma 3058 que la base documentaire «Jupiter» mentionne comme "plaque d'iconostase?", datée de l'Antiquité tardive.

25. Rapport de M. le Comte de Nieuwerkerke, surintendant des Beaux-Arts, membre de l'Institut sur les travaux de remaniement et d'accroissement réalisés depuis 1849 dans les Musées Impériaux suivi d'un relevé sommaire des objets d'art entrés dans les collections de 1849 à 1863, p. 48. 
d'appliques ${ }^{26}$. Dans la rubrique "Observations», nous trouvons l'indication "fouilles de Kertch", "trouvé à Kertsch» ou «Kertsch" ${ }^{27}$. Le livre d'entrée Napoléon III Mobilier de la Couronne II contient 183 numéros. Les bronzes sont également prépondérants avec 164 occurrences. On dénombre 62 boucles, 22 fibules, dont une en argent oxydé ${ }^{28}$, quelques bagues et anneaux, plaques de serrure, garnitures de serrure, un loquet, des disques, des clochettes. Les fibules, les clochettes, tout ce qui concerne les serrures et quelques disques sont indiqués comme provenant des "fouilles de Kertch". Le livre d'entrée N III ne mentionne que trois objets: une colonne arabe, une inscription et un devant de sarcophage. La colonne arabe et le devant de sarcophage sont expressément mentionnés comme venant de Crimée. Le tout est désigné comme étant un «don du Ministère de la Guerre ${ }^{29}$.

Une campagne de réinscription des objets, principalement ceux en bois, apparaît dans le Livre d'entrée $M N E$ : il s'agit d'un lot de 14 rondelles en bois et 17 autres objets, des pyxides et des couvercles essentiellement ${ }^{30}$. Ces 18 numéros relèvent selon les archives de l'«ancien fonds». Vingt numéros sont ensuite enregistrés le 14 juin $1985^{31}$. Il s'agit de la réinscription des stucs qui sont tous mentionnés comme venant de Kertch. Cet ensemble relève également de l'«ancien fonds».

Dans l'Inventaire S, ouvert en juillet 1918 par E. Pottier, conservateur au département, en vue d'y verser les pièces de la collection Campana et qui fut utilisé par la suite pour l'inscription de pièces qui ont perdu leur numéro d'inventaire, on ne mentionne expressément aucune pièce de la dévolution de l'armée d'Orient de 1856, ce qui est a priori normal. Cependant, un croisement opéré entre les numéros qui y figurent et la base documentaire "Jupiter", laquelle mentionne les objets provenant

26. Six de ces positions ne disposent d'aucune observation, alors que le reste est indiqué soit comme "fouilles de Kertsch" ou «trouvé à Kertsch». Bien que dépourvus de toute indication de lieu, ces objets ont été considérés comme provenant également de Kertch. De ce fait, ils sont inclus dans les statistiques (Livre d'entrée Napoléon III Mobilier de la Couronne I, p. 197 et 202).

27. Exemple: NAP III 860; N III 789, N III 900, N III 937; NAP III 1051, NAP III 1052, NAP III 1055.

28. NAP III 1891.

29. Livre d'entrée $N$ III, p. 94 positions 966, 967, 968.

30. Numéros du MNE 416 à MNE 432.

31. Numéros du MNE 876 à MNE 895. 
de cette dévolution, a permis d'en dénombrer 46 autres. Il s'agit principalement d'objets en bois: des pyxides et leurs couvercles, une statuette en bois dorée et peinte, ou encore des «fragments de décoration représentant des oves et rais-de-cœur peint rouge et or ${ }^{32}$.

Les difficultés à dénombrer l'ensemble des objets concernés sont multiples, du fait des différents critères qui entourent leur recensement dans les instruments de recherche disponibles au DAGER et que nous n'allons pas détailler ici. Par ailleurs, si on reprend le décompte fourni par Nieuwerkerke dans son rapport, il serait nécessaire de retrouver quelques-uns des 106 vases "en terre rouge" qui manquent encore à l'appel. On sait en effet que l'État organisa en 1875 l'envoi d'antiques dans les musées de province en ayant fait la demande et que les vases de Kertch furent ainsi intégrés à divers lots ${ }^{33}$. Restent ceux en verre et en albâtre, ainsi que les colliers, les figurines et les terres cuites. Ces dernières correspondent probablement aux appliques mentionnées dans la base "Jupiter" ${ }^{34}$. On y trouve encore des bronzes (fibules, boucles et éléments de serrure), ainsi que des bois de conservation très délicate. Les modalités présidant à l'intégration de ces objets aux collections du Louvre reflètent bien les pratiques d'une époque durant laquelle émerge progressivement la notion de conscience du patrimoine et de sa conservation, particulièrement en temps de guerre ${ }^{35}$.

32. S 2046.

33. Leur trace ne fut d'ailleurs retrouvée que très récemment. Ch. Orgogozo, Y. Lintz (dir.), Vases, bronzes, marbres et autres antiques, p. $27: 17$ verres et une caisse de 17 fragments de Kertsch; p. 58-60: collection de Kertsch et vases mis en dépôt; p. 123: pour dépôt à Draguignan; p. 130: pour dépôts à Tourcoing et Châteaudun; p. 131 : pour musée d'Orbec. Y. Lintz, «Les apports du récolement à la connaissance des collections». Discussion le 31 mars 2014 avec Mme C. Walter du service de Récolement des dépôts du musée du Louvre, et consultation de la base interne PICO.

34. MNE 876 à MNE 894.

35. La prise du Palais d'Été de Pékin, en 1858, constitue à ce titre un moment charnière, à en croire l'historien Alain Gouttman, qui considère comme significative la citation en justice des officiers pris à avoir voulu revendre des objets en provenant. 


\section{L'achat de 1920: la collection Messaksoudy}

\subsection{Le contexte}

Environ soixante ans plus tard, et malgré l'épisode de la tiare de Saïtapharnès, les objets provenant de Russie méridionale suscitent à nouveau l'intérêt des conservateurs du musée. La consultation rapide des catalogues de vente fait ainsi apparaître que la mention «Russie méridionale» ou "Crimée» est parfois mise en avant. Au début du XXe siècle, le marché de l'art parisien propose quelques ventes d'antiquités provenant de cette région. Si elles constituaient une infime partie des ventes archéologiques, elles ont cependant éveillé l'intérêt des collectionneurs ${ }^{36}$. Outre cela, la concurrence avec les musées allemands, toujours vivace en France, pousse alors les conservateurs français à investir cette aire géographique ${ }^{37}$. Le Louvre acquiert alors des antiquités provenant de Russie méridionale au gré des propositions de vendeurs et de marchands, comme le montre la chronologie des achats (fig. 1). Mais les acquisitions sont encore isolées et ne comptent pas de grandes séries.

L'occasion d'acquérir un important lot d'objets se présente en 1920, lorsqu'un collectionneur originaire de Kertch, Pierre Messaksoudy, propose à la vente sa collection d'antiquités. Elle rejoindra officiellement les collections nationales le 29 avril 1920, lorsque paraît au Journal officiel l'arrêté signifiant son achat par le ministre de l'Instruction publique et des Beaux-arts, André Honnorat. Ce texte administratif, banal et sec, ne rend pas compte des circonstances et des tensions que la proposition de cet achat a pu susciter au sein du musée du Louvre, ni de l'agitation qui a régné parmi les conservateurs et d'autres intervenants. Nous nous proposons de restituer la trame de cet achat hors du commun à l'aide de la documentation disponible tant aux Archives des Musées nationaux qu'aux Archives nationales et dans la documentation du DAGER.

36. Par exemple la vente Kibaltchitch en 1905 ou la vente de la collection Atrpet en 1908.

37. Notamment l'acquisition par les musées allemands de la collection Alexandre Merle de Massonneau en 1907 et en 1913, ainsi que la vente A. L. Vogell en 1908. 


\subsection{La famille Messaksoudy}

La famille Messaksoudy, d'origine grecque, s'est établie à Kertch vers 1860. Lancêtre Yani (Yannis/Ivan) y faisait commerce du vin. Son fils Konstantin Ivanovič devient fabricant de tabac et fournisseur de la cour des tsars ${ }^{38}$. Il ouvre son établissement en 1867 et exporte ses produits à Saint-Pétersbourg, Moscou, Kiev, Kharkov et d'autres grandes villes de l'Empire. Remportant plusieurs prix lors de différentes expositions en Russie, il s'enrichit rapidement et amasse une fortune considérable. C'est dans un contexte agité que Pierre Messaksoudy, un des fils de Konstantin, est nommé directeur du musée de Kertch le 2 janvier 1919. Le précédent directeur du musée, V. V. Škorpil, avait été assassiné par des fouilleurs clandestins, dans un contexte de guerre civile qui ravageait la Russie et qui s'avérait particulièrement féroce en Crimée.

Au printemps 1919, Pierre Messaksoudy et sa famille sont contraints de quitter la Russie, mais parviennent à emporter leurs biens, dont une prestigieuse collection d'antiquités. Il nous est difficile de restituer la trajectoire suivie par la famille Messaksoudy après son arrivée en France ${ }^{39}$. Les Archives de Police conservent la première demande de titre de séjour de Pierre, mais la date en est malheureusement illisible ${ }^{40}$. La correspondance échangée entre le marchand d'antiquités Henri Leman et Wilhelm Fröhner, ancien conservateur du musée du Louvre et personnage

38. Les informations concernant la famille ont été aimablement communiquées par M. Wladimir Messaksoudy, fils de Vassili et petit-neveu de Pierre Messaksoudy. Interview réalisé par mes soins le $1^{\text {er }}$ novembre 2013 à son domicile. Aussi N. V. Bykovskaja, "Grečeskaja obščina v èkonomičeskom razvitii Kerč-Enikal'skogo gradonačal'stva v konce XVIII - načale XX veka», p. 54 sq. et N. V. Bykovskaja, V. F. Sanžarovec, «Kerčenskij tabačnij fabrikant Konstantin Mesaksoudi». Au sujet de

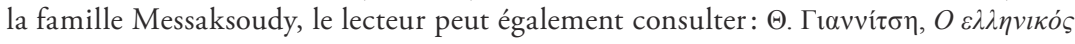

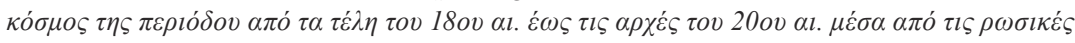

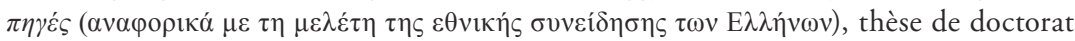
sous la direction de T. Nikitina, Université Lomonossov de Moscou, 2000, p. 119-146.

39. Les Archives des Douanes françaises ont été questionnées pour vérifier si l'arrivée de la famille Messaksoudy avec sa collection avait été signalée, mais rien n’apparaît dans les documents.

40. Service de la Mémoire et des Affaires Culturelles de la Police, Fichier étrangers sur microfiches, $\mathrm{n}^{\circ}$ 279, Demande numéro 141280 S, au nom de Messaksudy. Cette demande indique sa date de naissance ( $1^{\text {er }}$ février 1875$)$ à Kertch, sa nationalité hellène et donne les noms et les dates de naissance de ses parents sous la forme francisée: Constantin et Marie. D'autres fiches concernant la famille sont conservées dans ce fichier. 
incontournable du marché de l'art parisien en ce début du XXe siècle, évoque cependant l'arrivée d'une collection d'antiques provenant de Crimée le 16 juin 1919. Il est probable qu'il s'agisse de la collection Messaksoudy, puisque aucune autre vente d'antiquités de cette région n'a alors lieu, à notre connaissance. Par ailleurs, dans son Journal, Fröhner mentionne avoir vu la collection d'antiques de Kertch en date du 21 juin $1919^{41}$. On peut donc en déduire que Pierre Messaksoudy est arrivé à Paris au mois d'avril ou au mois de mai 1919 et a réussi à faire savoir au «tout Paris» des antiquaires qu'il avait une grande collection d'antiquités à vendre.

\subsection{Constitution de la collection en Russie}

Une partie de la collection de Pierre Messaksoudy provient de fouilles qu'il avait ordonnées sur ses terres ${ }^{42}$, le reste d'achats menés soit directement auprès de paysans ${ }^{43}$, soit auprès d'autres collectionneurs de Kertch ou d'ailleurs en Russie. Une partie de la collection avait ainsi été acquise auprès d'un collectionneur - de Bock ${ }^{44}$-, une autre - la plus prestigieuse - provenait de la collection du docteur Ignacy Terlecki, un officier des garde-côtes et médecin-chef de l'hôpital, dont les qualités de collectionneur étaient bien connues à Kertch ${ }^{45}$. La constitution de la collection

4I. Goethe und Schiller Archiv (GSA), Weimar, Allemagne: GSA 107/452, Fonds Fröhner, Lettres d'H. Leman, lettre en date du 16 juin 1919 et GSA 107/823, Fonds Fröhner, Journal de W. Fröhner (1915-1922), p. 121, en date du 21 juin 1919.

42. M. Kazanski, F. Beck, F. Vallet, "La riche tombe de Kertch du Musée des Antiquités Nationales", p. 63 et Mme T. V. Umrikhina, directrice du musée de Kertch dans un courrier qu'elle a bien voulu m'adresser.

43. Exemple dans l'inventaire MND no 1296 avec l'observation «apporté par des paysans» ou MND no 1331 "apporté par des paysans de Kertch".

44. V. N. Borovkova, Kollekcionery i torgovcy kerčenskimi drevnostjami, p. 35 sq. L'auteur indique qu'Alexandre Osipovitch Bock était un militaire, ingénieur-major en retraite, très actif dans l'administration municipale et d'autres institutions à Kertch. En 1911, J. J. Marti publia les inscriptions de la collection de Bock, alors que Mme de Bock offrait au musée de Kertch une stèle funéraire trouvée en 1912. D’autres objets furent légués au musée du kourgane du Melek-Tchesmé cette même année. Leur fils, Boris Alexandrovitch de Bock, hérita de la collection de son père et vendit les objets à P. Messaksoudy. Elle contenait des verres, des bronzes, de la céramique. Les de Bock procédaient à des fouilles sur leurs terrains.

45. Selon N. F. Fedoseev, Kerčenskij muzej drevnostej, p. 14, Terlecki constitue une première collection numismatique dont 829 objets furent vendus à la vente aux 
Messaksoudy est décrite également par Mikhail Ivanovič Rostovtzeff dans une note manuscrite, conservée aux archives du DAGER:

[...] Ce fut alors Messaxoudi qui en se guidant de conseils du feu prof. Škorpil [...] a acquis la coll. Terletzki. Après cette acquisition Messaxoudi n'a pas cessé d'augmenter la collection. N'étant pas connaisseur lui-même il consultait toujours le prof. Škorpil un savant éminent qui connaissait très bien l'archéologie de la Russie méridionale et qui lui-même dirigea pendant à peu près vingt ans les fouilles officielles à Kertch et dans les environs [...]. Comme M. Messaxoudi riche fabricant de tabacs était presque la seule personne qui disposait de fonds nécessaires pour acquérir ces objets trouvés il s’accapara par l'achat de la plupart des objets trouvés. Škorpil l'aidait car il était convaincu que Messaxoudi la crise russe passée vendrait ou léguerait sa collection à l'État russe. Après la mort de Škorpil Messaxoudi qui lui succéda comme directeur du Musée procéda toujours à enrichir sa collection privée $[\ldots]^{46}$

\subsection{Lacquisition de la collection par le Louvre}

Comment se déroule l'acquisition de cette collection par le musée du Louvre? ${ }^{47}$ Quelques pistes peuvent être envisagées, sans certitude.

enchères à Vienne en 1902, accompagnés d'un catalogue. Elle est achetée par un collectionneur de Moscou, F. I. Prowe, qui l'a revendue ensuite à l'étranger. Après la Révolution de 1917, une partie de la collection s'est retrouvée en Pologne: en 1925 et 1930, le Musée national de Varsovie a pu acquérir respectivement 815 et 936 monnaies, notamment celles du Bosphore Cimmérien et des colonies grecques de la mer Noire. Le Musée national de Cracovie avait déjà acquis quelques objets en 1908 (A. Szemiothowa, Numizmatyka starożytna et "Historia powstania działu numizmatyki starożytnej Muzeum narodowego w Warszawie», p. 341 sqq. Aussi J. Bodzek, "Coins from Greek Cities on the Northern Coast of the Black Sea in the Collection of the National Museum in Cracow», p. 66).

46. DAGER, Dossier Messaksoudy, sans cote.

47. Le déroulé de l'achat a été reconstitué par nos soins à partir de dossiers et procèsverbaux suivants: AMN : $2 \mathrm{NN}^{22}$ 1920, pièce $\mathrm{n}^{\circ} 27$ : $1^{\text {er }}$ payement à M. Messaksoudy sur 425.000 francs. Chapitre IV $V^{12}$ (175.000 francs); AN : F/21/ 4451 Achat Messaksoudy; AMN : *1 BB 39 Compte-rendu du Comité des Conservateurs, séance du Mercredi 24 mars 1920; AMN : *1 BB 39 Procès-verbaux de séances tenues par le Comité des Conservateurs, séance du lundi 19 avril 1920; AMN : ${ }^{*} 1$ BB 39 Procès-verbaux du Comité Consultatif. Séance du 6 mai 1920; AMN : *1 BB 39 Procès-verbal du Comité des Conservateurs du 6 janvier 1921; AMN : *3 BB 2 Procès-verbaux du 


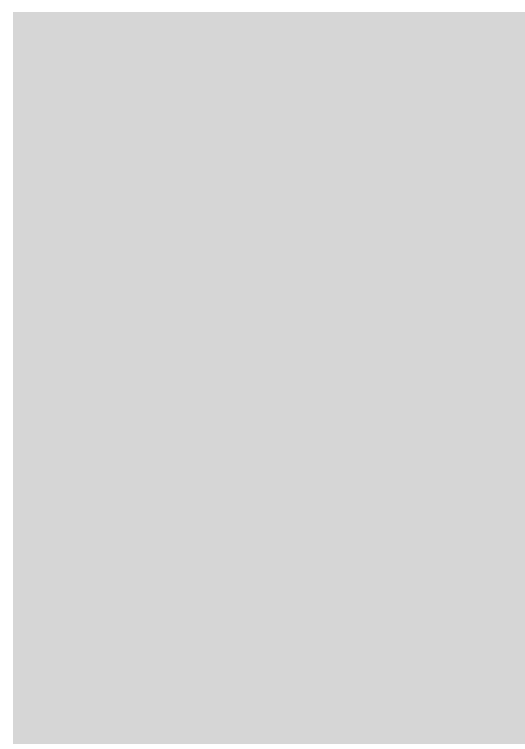

Fig. 2 - Portrait de Salomon Reinach (C) MAN, centre des archives.

Nous savons tout d'abord que M. I. Rostovtzeff est à Paris en $1920^{48}$ et qu'il a pu jouer le rôle d'intermédiaire. Le savant russe connaissait Salomon Reinach, directeur du Musée des Antiquités nationales de Saint-Germain-enLaye (M.A.N.) (fig. 2), avec lequel il entretenait une correspondance professionnelle et personnelle, comme en témoignent les lettres conservées à la Bibliothèque Méjanes ${ }^{49}$.

Le Journal rédigé par Edmond Pottier, à l'époque conservateur du département des antiquités orientales et de la céramique antique du musée du Louvre, pourrait constituer une autre piste ${ }^{50}$.

Quoi qu'il en soit, la collection russe - conservée dans un coffre-fort au Crédit Lyonnais, boulevard des Italiens - est examinée par E. Pottier le vendredi 19 mars 1920, après que ce dernier a consulté Rostovtzeff. Ce moment coïncide probablement avec la rédaction de sa note sur la collection Messaksoudy ${ }^{51}$.

À la lumière de la chronologie établie, la première mention officielle concernant la collection formée par Pierre Messaksoudy apparaît le 23 mars 1920, dans un courrier invitant les membres du Conseil des

Conseil artistique de la Réunion des musées nationaux 9 janvier 1911 - 7 novembre 1927, (numérisé) : Séance extraordinaire du mardi 20 avril 1920, p. 210 à 213. Documentation DAGER, Dossier Messaksoudy, sans cote.

48. Les registres des visiteurs du Cabinet des Médailles sont une autre source pour confirmer la présence de Rostovtzeff à Paris.

49. Bibliothèque Méjanes, Inventaire correspondance de S. Reinach, boîte $\mathrm{n}^{\circ} 145$. Sans cote.

50. Il s'agit d'un brouillon dans lequel E. Pottier décrit jour après jour les évènements et les rencontres qu'il a eus à propos de l'achat de la collection Messaksoudy. Ainsi Pottier note dans ce Journal à la date du 6 mars 1920: «visite au Louvre de Mlle Alia Berson Bertseff [...] de la part de M. et Mme Messaksoudy 4 av. de la Bourdonnais».

5I. Cf. supra, n. 49. 
Musées à se réunir exceptionnellement dans le hall du Crédit Lyonnais à Paris, le vendredi suivant (réunion qui sera finalement avancée de vingtquatre heures) ${ }^{52}$. Un courrier du directeur des Musées nationaux avait été précédemment envoyé à Pierre Messaksoudy qui en accuse réception le 23 mars et annonce sa venue au Crédit Lyonnais pour le lendemain. Après examen de la collection le mercredi 24 mars, le Comité des Conservateurs se réunit au Louvre pour délibérer. Le vendeur propose la collection sous forme d'un achat en bloc, pour la somme de 450 ’000 francs, une réponse étant exigée sous huit jours.

Trois conservateurs sont intéressés par cette collection et souhaiteraient acquérir des objets pour leurs départements et musées respectifs: Edmond Pottier pour le département des antiquités orientales et de la céramique antique du Louvre, Étienne Michon, conservateur en chef au musée du Louvre du département des Antiquités grecques et romaines et Salomon Reinach, directeur du Musée des Antiquités nationales de Saint-Germain-en-Laye. Chacun d'entre eux présente ses choix:

[E. Pottier souhaiterait réserver un] certain nombre de pièces céramiques. M. Michon voudrait réserver de son côté un vase en verre à frise peinte, une coupe en millefiori, [...], une cruche à panse décorée de pois bleus [...], cinq épingles à tige de bronze et tête de verre, une coupe en verre gravé à inscription et une niké en os. M. Reinach, pour sa part, souhaiterait acquérir un certain nombre d'objets provenant d'une tombe trouvée à Kertch, qui présentent un intérêt tout particulier: des plaques d'émail d'une technique tout à fait comparable, qui formaient des côtés d'une pyxide [...], une couronne faite de feuilles d'or portant l'empreinte de monnaies de Pupien [...]. La tombe a fourni également une grande épée à poignée d'argent, et dont la garde est constituée par un jade chinois ${ }^{53}$.

Lors de la délibération, Edmond Pottier souligne encore que «l'importance du prix demandé rend impossible l'acquisition, en bloc, de la

52. Le fonctionnement de la Réunion des musées nationaux prévoit que le Comité consultatif des conservateurs propose l'achat de pièces, le Conseil artistique le discute et ce n'est qu'après l'aval du Conseil, et sa proposition au ministre de l'Instruction publique et des Beaux-Arts, que l'achat, et donc l'inscription sur les livres d'entrée, peut s'effectuer. La lettre en question est si vite dactylographiée qu'il y a erreur sur le jour de la séance: à la place du vendredi initial a été inscrit jeudi.

53. AMN *1 BB 39: Compte-rendu du Comité des Conservateurs, séance du mercredi 24 mars 1920 , p. 3. 
collection, ou, du moins, impossible, l'obligation de ne conserver qu'une partie de collection. Mais, comment se défaire du reste?» ${ }^{44}$. La question est cruciale! Il faut non seulement vendre la partie que les conservateurs ne souhaitent pas conserver, mais aussi le faire avec prudence, car quelques pièces semblent fausses. Et cette publicité n'est évidemment pas souhaitée: la pénible affaire de la tiare de Saïtapharnès se rappelle aux souvenirs de tous, puisque les protagonistes participant à l'achat de la collection Messaksoudy y ont été mêlés. Dix-sept ans après l'établissement de la falsification, il n'est pas question de répéter l'erreur. Durant la même séance, E. Pottier propose d'acquérir les pièces de la collection qui intéressent les musées du Louvre et de Saint-Germain-en-Laye et de revendre le reste soit à un musée américain, soit sur le marché de l'art. Ce projet est avalisé par le Comité des Conservateurs qui le soumettra à l'unanimité au Conseil des Musées lors de sa réunion du vendredi 26 mars. On songe alors à avertir Paul J. Sachs, futur directeur associé du Fogg Art Museum, le plus ancien des musées de l'Université Harvard, de passage à Paris.

La collection doit être acquise pour la somme imposante de 450 '000 francs, ce que le Louvre ne peut se permettre ${ }^{55}$. Lors de la délibération du 24 mars, les trois conservateurs ont estimé de façon sommaire les montants liés à leurs choix: ainsi, la céramique grecque vaudrait environ de 60'000 francs selon E. Pottier; pour les Antiquités grecques et romaines, le coût reviendrait entre $22^{\prime} 500$ et $23^{\prime} 000$ francs et $\mathrm{S}$. Reinach avance la somme de $70^{\prime} 000$ francs pour un lot revenant au Musée de Saint-Germain-en-Laye.

[...] il resterait alors 250'000 à 300'000 francs à payer pour l'acquisition du reste par le Musée de Boston [soit le Fogg Art Museum, note de l'éditeur]. Comme celui-ci obtiendrait, à ce prix, très diminué,

54. Le site internet de l'Institut national de la statistique et des études économiques (INSEE) propose un convertisseur des francs en euro, suivant l'année de la monnaie d'origine et la conversion en euros de l'année 2012. Un calcul rapide permet d'avancer la somme de 421'026,35 euros. Source: <www.insee.fr/fr/themes/calcul-pouvoirachat $>$. En outre, ce prix de 450'000 francs pouvait représenter presque un quart des dépenses annuelles sur la ligne budgétaire IV ${ }^{12}$ "Acquisitions du Conseil des Musées» qui s'élevait, pour l'année 1920, à 1'807'981,75 francs (source: AMN 2 NN 22 1920, Rapport au Ministre de l'Instruction Publique et des Beaux-Arts du 6 juin 1921, p. 1).

55. A. Callu, La Réunion des Musées nationaux, p. 290. 
pour lui, encore une fois, du fait d'un change favorable, le quatre cinquièmes de la collection, et que, d'ailleurs, sa part (160 pièces environ sur 200) constituerait un ensemble comprenant, notamment, beaucoup d'objets en or, il semble que l'affaire se présenterait, pour lui, dans des conditions très avantageuses ${ }^{56}$.

Voilà donc un plan élaboré pour satisfaire tout le monde: le musée du Louvre - parce qu'il serait possible de constituer une collection où :

[...] dans aucun musée en dehors de la Russie, on ne pourra, mieux qu'au Louvre, étudier cette civilisation si curieuse importée par les Grecs dans leurs colonies de l'Europe [...] et ils [les trois conservateurs] se résigneraient difficilement, d'ailleurs, à rendre au vendeur les objets capitaux, la coupe de faïence figurant l'Atalante et la boite en émail [...] qui feront l'honneur de leurs départements et que déjà ont admirés ceux de leurs collègues étrangers à qui on a eu l'occasion de les présenter $[\ldots]^{57}$

et le musée de l'Université Harvard - parce qu'il aurait l'occasion d'acquérir à bon prix des objets en or. Il reste deux choses à faire: convaincre le Conseil des Musées nationaux et le représentant du musée américain.

Lachat de la collection en bloc représente en effet le nœud du problème. Lors de la délibération du 26 mars 1920, le Conseil fait sienne la proposition d'E. Pottier: acheter tout en bloc et revendre ce qui n'intéresse pas les conservateurs. Mais la question de la légalité d'une telle opération se pose. Un des membres du Conseil des Musées, R. Koechlin, imagine alors une autre solution: "un "ami" du Louvre qui se substituerait au musée pour le faire ${ }^{58}$. Solution tout à fait naturelle pour ce cofondateur, puis président, de la société des Amis du Louvre. Cette suggestion est acceptée par le Conseil qui décide de solliciter les bons offices de Maurice Fenaille. Celui-ci se laisse convaincre puisqu'il adresse

56. $\mathrm{AMN}{ }^{*} 1 \mathrm{BB} 39$ : Compte-rendu du Comité des Conservateurs, séance du mercredi 24 mars 1920 , p. 4.

57. AMN *3BB 2: Procès-verbaux du Conseil artistique de la Réunion des Musées nationaux 9 janvier 1911 - 7 novembre 1927, p. 213 et AN, F/21/4451 Achat Messaksoudy. Pièce: Extrait de la séance tenue le 26 mars 1920 par le Conseil des musées, p. 3.

58. AN, F/21/4451 Achat Messaksoudy. Pièce: Extrait de la séance tenue le 26 mars 1920 par le Conseil des musées, p. 2. 
le 2 avril une lettre manuscrite, sans que le destinataire soit nommé, indiquant qu'il va « remettre à M. Galbrunn la somme de 250'000 franc pour que vous en disposiez comme il a été convenu " ${ }^{59}$. Un reçu conservé dans les Archives des musées nationaux en date du 2 avril 1920 confirme ce versement ${ }^{60}$.

\subsection{Le musée américain}

E. Pottier note le lundi 29 mars 1920 : «Visite de M. Sachs au Louvre. Visite avec lui chez M. Gonse à 5 h" ${ }^{61}$. P. J. Sachs n'étant pas sûr de lui, il demande à revoir la collection. Cette nouvelle visite a lieu le mercredi 31 mars en compagnie d'E. Pottier et de Mlle Hetty Goldmann, archéologue. Manifestement M. Sachs et Mlle Goldmann semblent convaincus. E. Pottier note: "Sachs va faire un câble pour New York ou à Boston pour l'acquisition du lot de 250.000. Réponse sous 4 ou 5 jours» ${ }^{62}$.

Le $1^{\text {er }}$ avril 1920, on prépare la lettre à M. Misaksoudis [sic] indiquant que:

[...] les Commissions compétentes des Musées Nationaux ont examiné la collection que vous aviez proposée à l'acquisition au prix de quatre cent vingt-cinq mille francs. Elles se sont mises d'accord pour accepter

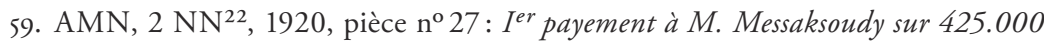
francs. Chapitre IV 12 (175.000 francs).

6o. Les échéances sont prévues pour le 28 août (10’000 fr.), 3 octobre (110’000 fr. et 5’000 fr.) et 7 octobre ( 130 '000 fr.) soit un total de $255^{\prime} 000$ francs.

6I. Journal d'E. Pottier.

62. Le "câble» de Paul J. Sachs est conservé aux archives de Harvard Art Museums. Il s'agit en réalité d'une très longue lettre de plusieurs pages, écrite sur papier à en-tête de l'hôtel Lutetia. Sachs y évoque ses pérégrinations dans la recherche d'œuvres d'art pour le compte du Fogg Art Museum. Concernant la collection Messaksoudy, il rend compte de la proposition faite d'acquérir la part abandonnée par le Louvre. Son regard s'avère très lucide dans la mesure où il a parfaitement saisi le sens de la démarche des conservateurs qu'il ne semble pas avoir appréciée. Lettre de Paul J. Sachs à Edward Waldo Forbes, Mars 31, 1920. Edward Waldo Forbes Papers (HC 2), file 1869. Harvard Art Museums Archives, Harvard University, Cambridge, MA. Je souhaite remercier Mesdames Susanne Ebbinghaus, conservateur au département de l'art antique aux Harvard Art Museums, Megan Schwenke, archiviste/gestionnaire et Brooke McManus, assistante archiviste aux Harvard Art Museums Archives pour leurs recherches concernant la lettre de Paul. J. Sachs. 
le prix proposé. [...] $1^{\circ}$ qu'une somme de 175.000 f vous sera payée par voie de mandat sur la Caisse des Musées selon les règles en usage, $2^{\circ}$ qu'une somme de $250.000 \mathrm{f}$ vous sera payée par une tierce personne, la réunion de ces deux sommes réglant ainsi le prix convenu de l'ensemble de la collection ${ }^{63}$.

Entre-temps, une diminution de la somme à verser à Pierre Messaksoudy est négociée: de 450'000 francs dont il a été question jusque-là, on passe à $425^{\prime} 000$ francs. Une diminution de $25^{\prime} 000$ francs est accordée en raison de l'existence de pièces jugées fausses par les conservateurs. Le lundi 5 avril, Paul J. Sachs annonce sa réponse:

Je regrette vivement que l'impossibilité d'avoir l'argent disponible m'empêche de faire l'acquisition des objets grecs. Soyez assuré de ma profonde reconnaissance pour votre amabilité que je n'oublierai pas et recevez l'assurance de mes sentiments très dévoués. Paul J. Sachs ${ }^{64}$.

C'est un refus inattendu qui surprend: il faut donc monter une nouvelle stratégie. L. Gonse fait tout de suite une autre proposition pour la vente du reste de la collection:

[...] il va falloir se retourner autrement. Mais puisque la Caisse a le droit d'acquérir et de vendre, la vente de la collection Missaxoudis [sic] ne pourrait-elle être faite sous son couvert par les soins de la Conservation? Ce ne serait pas le Musée du Louvre, mais la Caisse, personnalité civile qui vendrait ${ }^{65}$.

Malgré ce retournement de situation et l'incertitude qui plane désormais sur l'achat, le directeur des Musées nationaux prépare le mercredi 7 avril un courrier au ministre de l'Instruction publique et des Beaux-Arts concernant l'acquisition de la collection Messaksoudy.

Lors de son exposé devant le Comité des Conservateurs le 24 mars, E. Pottier s'était refusé à vendre la partie qui n’intéressait pas les

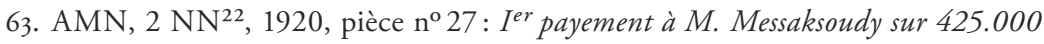
francs. Chapitre IV 12 (175.000 francs). Pièce: Paris, le 1 avril 1920 M. Misaksoudis 4 av. de la Bourdonnais.

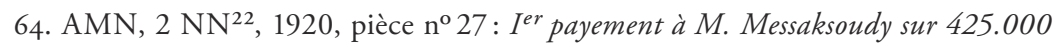
francs. Chapitre IV $V^{12}$ (175.000 francs). Pièce: Télégramme de Paul J. Sachs.

65. AMN, $2 \mathrm{NN}^{22}, 1920$, pièce $\mathrm{n}^{\circ} 27$ : I ${ }^{\text {er }}$ payement à M. Messaksoudy sur 425.000 francs. Chapitre IV $V^{12}$ (175. 000 francs). Pièce: Lundi 6 avril, Mon cher Galbrund [sic]. 
conservateurs à des marchands. Les conditions ayant changé, il allait désormais falloir réfléchir à cette solution. Pottier note ailleurs dans son Journal avoir rencontré Fernand Lair-Dubreil, commissaire-priseur à Drouot. Il sollicite également le concours de marchands bien connus tels Feuardent et Leman dont les livres d'entrée du DAGER mentionnent de nombreuses acquisitions antérieures ${ }^{66}$. Lundi 12 avril, le Conseil des Musées se réunit à nouveau:

M. le directeur fait savoir que M. Fenaille consent d'acquérir, pour le prix de $250.000 \mathrm{f}$ le second lot que le musée ne croit pas devoir acheter et qu'il a remis un chèque de cette somme. Il le fait parce que le vendeur s'est refusé à céder sa collection autrement qu'en totalité et en vue de rendre service au musée. Ce lot, qui n’a pas pu être vendu à un musée américain, comme on l'espérait, sera mis en vente à l'Hôtel Drouot par les soins des conservateurs compétents qui en dresseront le catalogue, sans que le nom de M. Fenaille y soit inscrit: la vente faite, le produit sera versé à $M$. Fenaille, la Caisse des Musées s'engageant à compléter la somme de 250.000 francs si elle n'était pas atteinte et $\mathrm{M}$. Fenaille à ne pas demander une part dans le bénéfice s'il en était réalisé un. [...]. La discussion s'engage sur les modalités de l'opération ${ }^{67}$.

Mardi 13 avril 1920, Messaksoudy signe le reçu sur papier à en-tête des Musées nationaux.

3.6. La note de François Thiébault-Sisson et la réponse de la Réunion des musées nationaux

Quelques jours plus tard, François Thiébault-Sisson adresse une note au bureau du directeur des Musées nationaux, mettant en garde les Musées contre l'achat de faux. Le journaliste et critique d'art, régulièrement

66. Pottier le note dans son Journal à la date du samedi 10 avril: «Vu M. Leman et Feuardent ». Pour Leman le Livre d'entrée MNB 1871-1881 indique les numéros MNB 351 et 352, p. 63-64 (en 1872) ou MNB 649 p. 113-114 (en 1874). Pour Feuardent environ 200 positions sont recensées, selon A. Pasquier, «Une nouvelle acquisition du musée du Louvre».

67. AN, F/21/4451 Achat Messaksoudy. Pièce: Extrait de la séance tenue le 12 avril 1920 par le Conseil des musées. 
publié dans le journal Le Temps depuis $1880^{68}$, provoque un branlebas de combat: le scandale de la tiare de Saïtapharnès, couvert par la presse jusqu'en 1903, est encore dans tous les esprits ${ }^{69}$. Or les allégations formulées dans sa note par F. Thiébault-Sisson - qui n’avait lui-même pas vu les objets, mais s'en remettait à un informateur - s'apparentaient davantage à un règlement de compte visant E. Pottier.

Thiébault-Sisson était l'auteur de billets au vitriol qui frisaient la calomnie. Il y dénonçait «le gaspillage des finances d'un musée dont l'ignorance et le défaut de prévision sont une proie pour des commerçants avisés, admirablement informés et qui excellent non seulement à prévoir, mais à solliciter adroitement l'occasion", poursuivant sur l'incompétence du personnel "toujours recruté au hasard et dont on n'exige, en le nommant, aucune preuve d'aptitudes réelles et de savoir spécial", ce qui expliquait "à merveille les erreurs commises par le Louvre, et le conseil des musées $[\ldots]{ }^{70}$.

Le ton tout aussi provocateur de sa note, intitulée "Une nouvelle affaire de la tiare au Musée du Louvre», justifia que le Comité des Conservateurs se réunît en séance extraordinaire de sorte à réfuter immédiatement les accusations du journaliste susceptibles de «jeter la suspicion du public sur la collection que les Musées nationaux viennent d'acquérir ${ }^{71}$. Pottier cita encore une fois l'appréciation de M. Rostovtzeff à l'appui de son propre point de vue, "l'un des meilleurs archéologues de la Russie du Sud, chargé de cours à Oxford et au Collège de France, qu'il a interrogé. [...]. Il se porte garant de l'honorabilité de M. Messaksoudy et estime l'ensemble exhumé par lui dans ses fouilles non seulement

68. Une requête rapide sur le site de la BNF Gallica (<http://gallica.bnf.fr> recherche "Thiébault-Sisson») fait apparaître 1185 résultats de publications dans le journal Le Temps, entre le 31 mai 1880 et le 12 juin 1938. Consultation le 6 mars 2014.

69. Une requête sur le site de la BNF Gallica (<http://gallica.bnf.fr > - recherche "Saïtapharnès») fait apparaître 4 résultats de publications dans le journal Le Temps, entre le 12 avril 1896 et le 27 septembre 1896, une en 1897 et environ une trentaine en 1903. Consultation le 6 mars 2014. Voir également l'interview accordé à ce propos par Salomon Reinach à Salomon Thiébault-Sisson dans Le Temps du 14 avril 1903, p. 4, article La tiare de Saïtapharnès.

70. Le Temps du 14 janvier 1912, p. 3, article La vie artistique.

71. AMN *1 BB 39: Compte-rendu du Comité des Conservateurs, séance du lundi 19 avril 1920, p. 51-56. 
indiscutable, mais du plus haut intérêt ${ }^{72}$, remportant l'adhésion définitive du Comité. Edmond Pottier et Salomon Reinach soulignent l'intérêt de la collection Messaksoudy pour les musées français:

[...] dans aucun Musée en dehors de la Russie, on ne pourra mieux qu'au Louvre, étudier la civilisation si curieuse importée par les Grecs dans leurs colonies de l'Euxin. Ils se résigneraient difficilement, d'ailleurs, à rendre aux vendeurs des objets capitaux $[\ldots]^{73}$

Le 7 juin 1920, le Conseil des Musées approuve l'acquisition de l'intégralité de la collection Messaksoudy pour la somme de $425^{\prime} 000$ francs - ce sera la seconde plus grosse dépense de l'année, après L'Atelier de Courbet (une toile acquise pour la somme de 700'000 francs). Le rapport annuel présenté au ministre de l'Instruction publique et des Beaux-Arts en 1921 est soumis le 6 juin et sera publié au Journal officiel à la date du 17 juin $1921^{74}$.

3.7. Composition de la collection et sa présentation dans les musées

Au milieu du tumulte provoqué par la note de F. Thiébault-Sisson, le 19 avril 1920, la part de la collection Messaksoudy destinée au musée du Louvre est officiellement inscrite sur le livre d'entrée $M N D$ du département des Antiquités grecques et romaines et un peu plus tard sur le livre d'entrée $C A$ des Antiquités orientales et de la céramique antique ${ }^{75}$. La part qui revient au Musée de l'Archéologie nationale de Saint-Germainen-Laye est inscrite sur son propre inventaire au mois de mai $1920^{76}$.

72. AMN *3BB 2: Procès-verbaux du Conseil artistique de la Réunion des Musées nationaux 9 janvier 1911 - 7 novembre 1927. Séance extraordinaire du mardi 20 avril 1920, p. 210-213.

73. Ibid.

74. Journal officiel du 17 juin 1921, p. 6931 sq.

75. Mais il s'agit en réalité d'une inscription postérieure: le 19 avril, le Comité des Conservateurs se réunit dans l'après-midi à 16 heures et le Conseil artistique se réunit le lendemain matin à 10 heures et quart. L'inscription a donc dû intervenir entre le 20 avril et le 29 avril, date de signature du décret par le ministre, et même, comme nous le soupçonnons, s'étaler sur plusieurs jours, vu le nombre de pièces. Voir pour cela A. Callu, La Réunion des Musées nationaux, p. 172.

76. M.A.N., Registre numérisé $n^{\circ} 8$, signets de 64027 à 64047 . Il est à noter qu’à l'époque le musée porte le nom de Musée des Antiquités Nationales. 


\subsubsection{Au Louvre}

La lecture du livre d'entrée $M N D$ montre que le nombre d'objets s'élève déjà à 295, sans prendre pour autant en compte un lot de bractées - dont la quantité est donnée dans la base documentaire "Jupiter" ${ }^{77}$ - portant ainsi l'ensemble des pièces à un total de 783. De ce nombre, il faut toutefois déduire quatre monnaies qui sont transmises au Cabinet des Médailles de la Bibliothèque nationale ${ }^{78}$, ainsi qu'une dizaine de vases en verre déposés après la Deuxième Guerre mondiale au département des Antiquités orientales ${ }^{79}$ et deux objets au département des Antiquités égyptiennes ${ }^{80}$. On arrive donc à un chiffre de 279 objets, exclusion faite des bractées.

Sous la houlette d'E. Pottier, l'inscription est opérée avec méthode, selon le matériau ou la dénomination. On inscrit ainsi d'abord les 73 vases en verre de formes diverses ${ }^{81}$. Viennent ensuite une victoire ailée en os, les 25 bronzes ${ }^{82}$, puis un fragment d'instrument en fer, 24 plâtres $^{83}$, les bijoux ${ }^{84}$ et la céramique ${ }^{85}$.

Dans la mesure du possible, la provenance de l'objet est également mentionnée. Il est ainsi possible d'identifier ce qui provient de l'ancienne collection Terlecki (18 entrées le signalent), ce qui a été trouvé sur les terres des Messaksoudy à Kertch, ce qui a été rapporté par des paysans ${ }^{86}$, ce qui a été racheté à des personnes privées ou encore ce qui provient de la région de Kertch, de Taman ou de Phanagorie.

77. MND 1283/Bj 67. La base documentaire «Jupiter» les répartit en cinq lots, d'un total de 488 pièces: elle distingue un lot de 20 pièces, un autre de 251 pièces, un troisième de 57 pièces, un autre encore de 117 pièces et enfin un dernier lot de 43 pièces.

78. MND 1343 à MND 1346. Déposés par décret du 26 février 1921.

79. En deux versements: 1948 et 1968. MND 1137, 1138, 1153, 1155, 1156, 1158, 1159, 1163, 1165, 1171.

8o. Versement en 1949, MND 1118 et MND 1125.

8I. La dénomination originale du livre d'entrée est conservée: alabastres, bouteilles, œnochoés, gobelets, amphorisques.

82. Statuette de Dioscure, lampes, strigiles, miroir, fibules, garnitures de trousse cinq épingles de bronze et de verre.

83. Représentant les masques de Méduse, masques de théâtre, des personnages divers.

84. Colliers, bracelets, couronnes, boucles d'oreille, bagues.

85. Coupes, amphores, diverses statuettes et lampes.

86. Exemple MND 1337/Bj 64 et $\mathrm{Bj} 65$. En tout, il y a sept mentions de ce type dans le livre d'entrée $M N D$. 


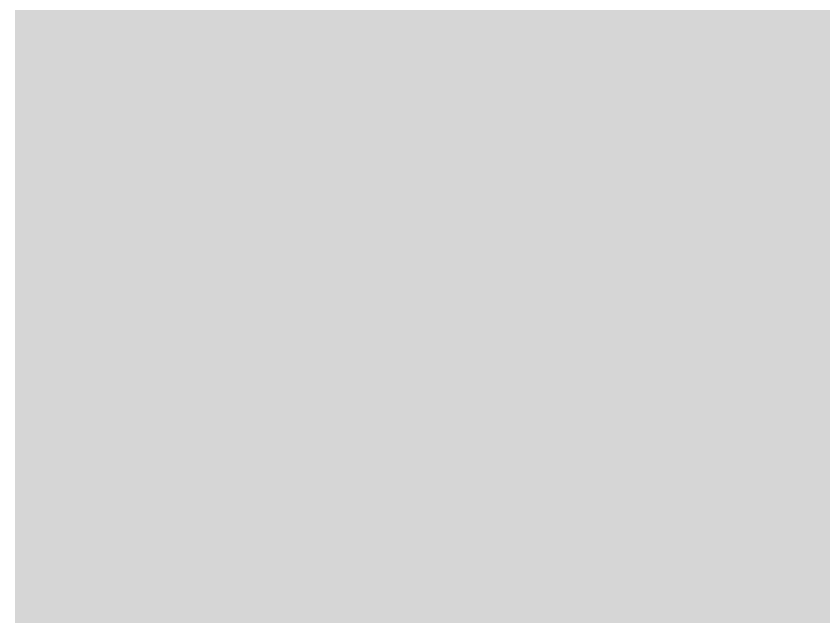

Fig. 3 - Collier à perles de cornaline, de montage moderne. Paris, musée du Louvre, MND 1236/Bj 631 @ RMN-Grand Palais (musée du Louvre) / Hervé Lewandowski.

Les bijoux

Forts de 161 pièces, auxquelles on ajoutera les bractées précédemment décrites ${ }^{87}$, les bijoux fournissent l'essentiel de la collection Messaksoudy. Leur inscription dans le livre $M N D$ est complétée par le numéro de catalogue $(\mathrm{Bj})$, qui possède sa logique propre de numérotation. Les colliers occupent à eux seuls 58 positions. Ils peuvent être composés de plusieurs éléments pouvant atteindre cinquante-huit pour le collier en cornaline acquis auprès du docteur Terlecki (fig. 3) ${ }^{88}$. D’autres matériaux sont également représentés: tel le verre ou la pâte de verre, et, plus précieux encore, l'or, la calcédoine, le cristal de roche, l'émeraude ou les grenats ${ }^{89}$. Deux sont d'emblée suspectés d'être «d'antiquité douteuse » ${ }^{90}$.

La deuxième place revient aux 32 boucles d'oreilles, toutes en or. Elles sont, pour la plupart, inscrites par paire, dont trois sont indiquées d'antiquité douteuse ${ }^{91}$. En ce qui concerne les 21 bagues, toutes sont

87. Bractées «trouvées à Kertsch par un Italien - avec la n ${ }^{\circ} 288$ - près de la propriété Messaksoudis» MND 1283/Bj 67.

88. MND 1236/Bj 631, ancienne collection Terlecki.

89. MND 1262/Bj 589; MND 1274/Bj 590; MND 1287/Bj 569.

90. MND 1285/Bj 499 et MND 1286 sans numéro Bj.

9г. MND 1289/Bj 313 et Bj 314; MND 1352/Bj 296 et Bj 297; MND 1353/Bj 352 et $\mathrm{Bj} 353$. 
en or, certaines avec intailles. On recense également 12 bractées ${ }^{92}$, en surcroît du fameux lot quantifié à part, façonnées dans la tôle d'or et certifiées authentiques. La collection comporte en outre sept bracelets en or, deux couronnes en or et deux feuilles de couronne séparées, exécutées également dans ce même matériau, cinq médaillons en or, cinq pendentifs et pendeloques, un anneau, des cylindres, un bouton, une valve de coquille, tous en or. D'autres objets sont encore recensés ${ }^{93}$.

Quatre monnaies antiques portant les effigies des rois du Bosphore ont été déposées au Cabinet des Médailles de la Bibliothèque nationale. Elles sont inscrites sur le Registre des dons $Y$ du Cabinet le 10 mars 1921, sous les numéros allant de Y 4269 à Y 4272. Il s'agit d'une monnaie d'Aspurgus, une de Sauromatès II et deux de Rheuscuporis III. La première est en or, les trois autres en électrum.

\section{Les bronzes}

Des bronzes sont également rapportés dans le livre d'entrée $M N D$ et portent en complément le numéro du catalogue $(\mathrm{Br})$. Ils sont au nombre de 25 et incluent deux poids en plomb. On y rencontre divers objets dont un Dioscure vêtu d'une chlamyde qui, tout comme les deux patères ou miroirs, est désigné comme appartenant à l'ancienne collection du docteur Terlecki ${ }^{94}$.

\section{Les verres}

On dénombre 73 entrées correspondant à autant d'objets en verre ${ }^{95}$. S’y côtoient: 22 flacons, 11 œnochoés, dix gobelets, six alabastres, cinq

92. MND 1333 à MND 1339 (les numéros $B j$ ne se suivent pas: on va du $B j 27$ à $\mathrm{Bj} 30, \mathrm{Bj} 55$ et 56 et ensuite du $\mathrm{Bj} 60$ à 65).

93. Petit vase sphérique et une spatule de cuiller en argent, deux lamelles rectangulaires, une en or l'autre en argent et deux fibules en or.

94. Quatre garnitures de trousse MND 1203 à MND 1206, cinq fibules, un miroir dont le conservateur était sûr et deux inscriptions: "patère ou miroir ", deux strigiles, une lampe, un fond de passoire, un anneau, deux fragments non identifiés et plusieurs fragments d'instruments, une hache à deux branches, un grand étui cylindrique, une cuiller. Le Dioscure porte le numéro MND 1186/Br 4126. Les deux miroirs portent les numéros MND 1187/Br 4127 et MND 1188/Br 4128.

95. MND 1111 à MND 1184. 


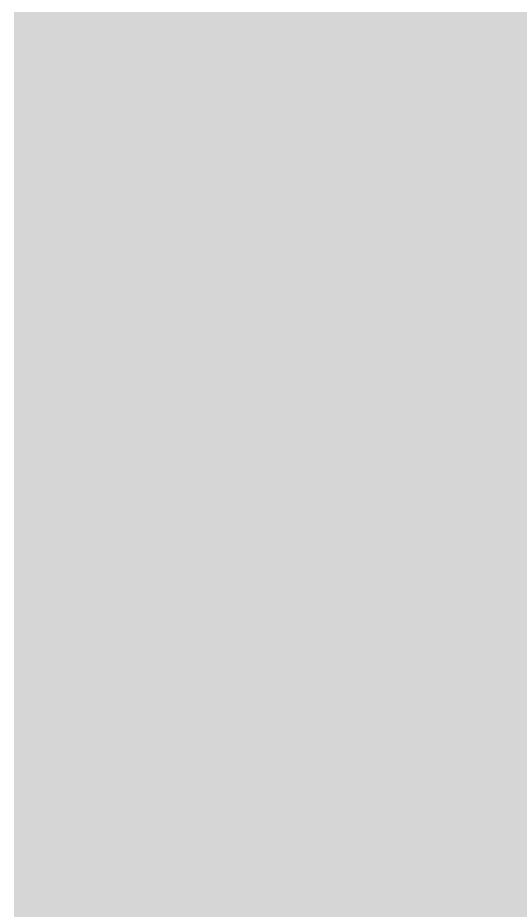

Fig. 4 - Grande cruche en verre à pastilles bleues de la collection Messaksoudy. Paris, musée du Louvre, MND 1177. (C) RMN-Grand Palais (musée du Louvre) / Hervé Lewandowski. coupes et cinq bouteilles, quatre amphorisques et quatre aiguières. À ce lot sont encore adjoints cinq épingles en bronze surmontées de têtes à double face ${ }^{96}$, un vase en forme de tasse, un petit bol en verre irisé et un plateau. Trois objets sont identifiés comme provenant de l'ancienne collection Terlecki dont une grande cruche avec anse ornée d'un réseau de filets et des pastilles ovales bleu foncé (fig. 4) ${ }^{97}$. La plupart des objets de ce lot sont réputés provenir de la région de Kertch. La totalité des objets mis en dépôt dans le département des Antiquités égyptiennes et le département des Antiquités orientales entre dans cette catégorie ${ }^{98}$.

Les stucs et autres matériaux

On trouve dans la collection Messaksoudy des objets qualifiés de "plâtres", c’est-à-dire des appliques en stuc ${ }^{99}$. Leur inscription est continue: bien que l'on relève 10 numéros sur le livre d'entrée, il y a en réalité 24 pièces conservées. Tous ces plâtres sont des représentations plastiques et viennent de la région de Kertch ${ }^{100}$.

96. MND 1183.

97. MND 1177, MND 1182 et MND 1184.

98. Ces objets ont été déposés en 1949, pour ceux qui concernent les Antiquités égyptiennes soit MND 1118 et MND 1125 et soit en 1948 soit en 1968, pour ceux des Antiquités orientales: MND 1153, 1155, 1156, 1158, 1159, 1163, 1165 et 1171.

99. MND 1212 à MND 1222.

IOo. C'est le cas du numéro MND 1213 qui mentionne "quatre masques de Méduse» ou encore celui du numéro MND 1215 «cinq masques tragiques". À propos des appliques de sarcophages, voir P. Pinelli, A. Wąsowicz, Catalogue des bois et stucs grecs et romains provenant de Kertch, ainsi que la contribution d'O. Samar dans ce volume. 


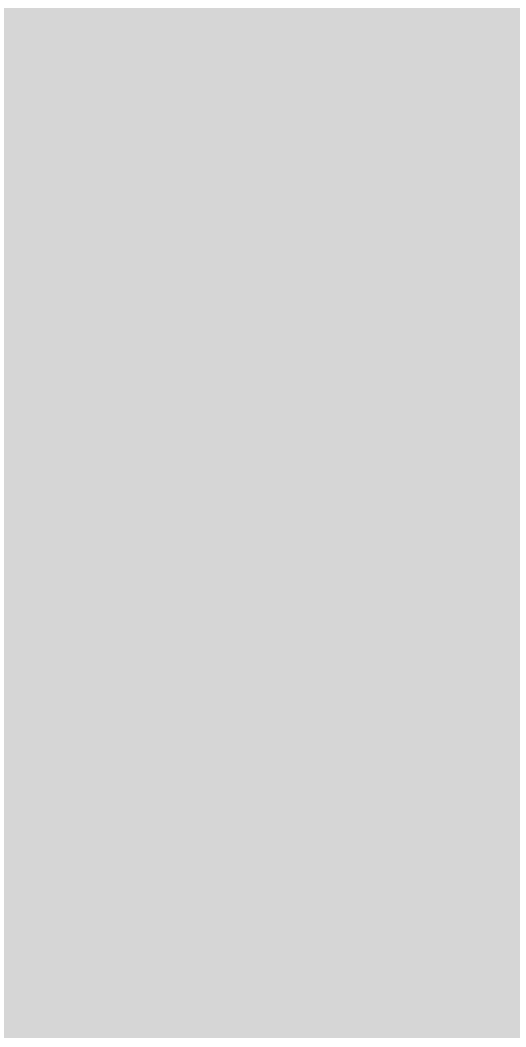

Fig. 5 - Victoire en os (hauteur $4 \mathrm{~cm}$ ). Paris, musée du Louvre, MND 1185. (C) RMN-Grand Palais (musée du Louvre) / Stéphane Maréchalle.
La partie de la collection inscrite dans le livre $M N D$ comporte encore des objets en os: une rondelle, un bouton circulaire, une spatule, des aiguilles, ainsi qu'une victoire ailée (fig. 5) ${ }^{101}$.

\section{La céramique}

La céramique est répertoriée dans le premier volume du livre d'entrée CA 1886-1968 (pour Céramique antique, appellation en vigueur à l'époque). Ici la date d'inscription est plus vague: avril-mai 1920 . On dénombre 64 inscriptions continues ${ }^{102}$. Elles ne sont pas aussi méthodiques que dans le livre d'entrée $M N D$. Quand la technique d'exécution l'exige, on précise qu'il s'agit de pièces à figures noires ou à figures rouges, mais cette indication n'est pas toujours fournie. Parmi les pièces de ce lot, la catégorie la plus importante regroupe 13 représentations plastiques en terre cuite, telle une Aphrodite anadyomène dans sa coquille (fig. 6) ${ }^{103}$. Viennent ensuite 11 coupes, entières ou fragmentées, dont le décor est éventuellement détaillé, comme dans le cas de la "petite coupe à l'Atalante» ${ }^{104}$. Des indications typologiques, voire techniques, sont parfois annotées, à l'instar d'un groupe de sept

IOI. MND 1185.

IO2. CA 2251 à CA 2314.

I03. Chaque pièce est décrite en quelques traits: «vieille femme grotesque à demi nue portant un vase» (CA 2295) ou "Vénus nue debout, dans une coquille» (CA 2288).

I04. CA 2259. La base documentaire "Jupiter" la mentionne actuellement comme «non-retrouvée». C'est l'un des objets convoités par les conservateurs du Louvre et décrits dans les Accroissements des musées nationaux. 


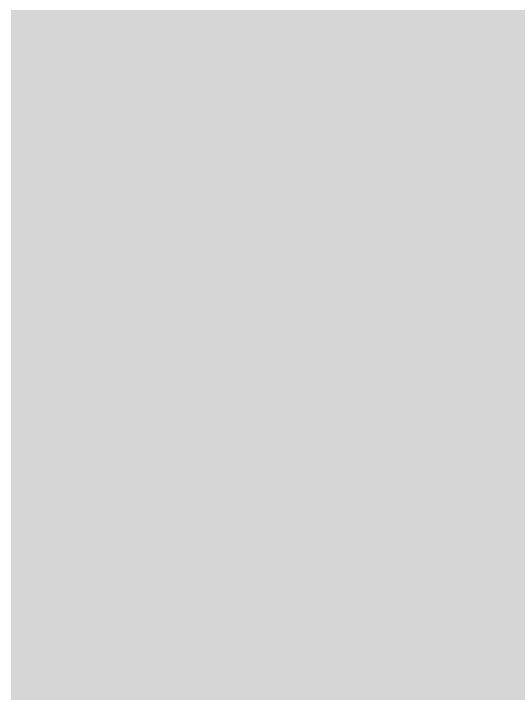

Fig. 6 - Statuette d'Aphrodite anadyomène dans une coquille (fin ${ }^{\text {er }}$ s. av. début $I^{\text {er }}$ s. apr. J.-C.) (hauteur $13 \mathrm{~cm}$ ). Paris, musée du Louvre, CA 2288. (C) RMN-Grand Palais (musée du Louvre) / Hervé Lewandowski.

amphores, régulièrement qualifiées "d'amphore-pélikè», mais ce point n'est pas systématique ${ }^{105}$. En revanche, la couleur de la pâte des six lampes est mentionnée - rouge, noire ou brune -, ainsi que la description du décor ${ }^{106}$. Il en va de même pour quatre lécythes ${ }^{107}$, trois masques et trois hydries ${ }^{108}$. Les bols mégariens sont caractérisés par leur décor à relief et les trois œnochoés portent des indications soit du matériau soit du décor ${ }^{109}$. La collection est complétée par une pyxide noire et d'autres objets encore $^{110}$.

3.7.2. Au musée de SaintGermain-en-Laye ${ }^{111}$

Le choix des objets acquis pour le musée de Saint-Germain-en-Laye est en rapport avec les collections du musée: l'accent est mis sur les objets mérovingiens et sarmates. Une notice de la main de Salomon Reinach en date du 29 mai 1920 précise que le lot d'objets de Kertch est extrait d'une collection plus importante

I05. Elles sont davantage caractérisées par le sujet représenté: «coureur dans la palestre» ou "femmes à la toilette» (CA 2252 et CA 2263).

Io6. CA 2302 à CA 2306 et CA 2309.

I07. CA 2254 à CA 2256 et CA 2266 qui toutes possèdent une indication de figures noires ou rouges et le décor qu'elles portent.

I08. CA 2299 à CA 2301 pour les masques et CA 2260, CA 2270 et CA 2272 pour les hydries.

I09. CA 2285 à CA 2287 pour les bols de Mégare et CA 2271, CA 2284, CA 2253 pour les œnochoés.

IIo. CA 2262. Petit amphorisque, un plat noir avec une salière au centre (CA 2275) deux canthares à anses en volute, un skyphos et des fragments de terre cuite à sujet érotique, buste féminin, ou fragment de vase avec masque comique.

III. M.A.N., Registre no 8 (59385 à 75968, signets 64027 à 64047), version numérisée. 
"formée en Crimée par le grec MISAXOUDIS acquise par le Conseil des Musées en avril-mai 1920 (425’000 fr.). Les objets attribués à SaintGermain sont estimés à 70'000 francs " ${ }^{112}$. La part de la collection Messaksoudy au Musée des Antiquités nationales est peu importante en comparaison de celle déposée au Louvre, mais c'est proportionnellement celle qui a coûté le plus cher - $100^{\prime} 000$ francs ${ }^{113}$.

L'ordre d'inscription au catalogue se fait ici en fonction de l'importance des objets: le premier numéro est attribué à l'épée en fer à poignée d'argent et garde de jade, le deuxième à la pyxide d'argent émaillée. Si 24 numéros d'inventaire sont attribués en tout, un numéro peut regrouper plusieurs objets; on totalise ainsi 57 objets distincts conservés à SaintGermain-en-Laye ${ }^{114}$. Les informations les concernant peuvent être très développées. Ainsi l'épée est-elle considérée dans le détail de ses matériaux constitutifs; l'opinion de M. Rostovtzeff au sujet de l'intérêt indiscutable du mobilier de Kertch pour l'art gréco-sarmate est également rapportée. Pour d'autres objets, comme la ceinture, la description technique est méticuleuse, allant jusqu'à préciser le nombre d'éléments qui la constituent ${ }^{115}$. Mais, ici aussi, le traitement du mobilier archéologique n'est pas standardisé et la description peut s'avérer parfois très succincte.

On sait «deux grosses boucles d'oreille» provenir de l'ancienne collection Terlecki ${ }^{116}$, quand certains objets sont "réputés faux", comme le vase en or repéré par les conservateurs, ou seulement "suspects» ${ }^{117}$. Par ailleurs, à la marge de l'inventaire, figure une information selon laquelle le 7 juin 1920 le musée de Saint-Germain a accueilli le "dépôt des objets d'or réputés faux compris dans l'acquisition Mesaxoudi "118. À quelques exceptions près, toutes ces informations sont reprises dans le Catalogue illustré du Musée des Antiquités Nationales au château de Saint-Germainen-Laye, à la page se référant à la collection Messaksoudy ${ }^{119}$.

II2. M.A.N., Registre numérisé no 8, signets de 64027 à 64047.

II3. AMN, $2 \mathrm{NN}^{22}$ Achat Messaksoudy $1^{\text {re }}$ partie, publication au Journal officiel du 17 juin 1921, p. 6932.

II4. No inv. de 66111 à 66134.

II5. $\mathrm{N}^{\circ}$ inv. 66113.

II6. $\mathrm{N}^{\circ}$ inv. 66127.

II7. Respectivement, $\mathrm{N}^{\circ}$ inv. 66130 et $\mathrm{N}^{\circ}$ inv. 66128.

II8. Page 359 de l'inventaire, note en bas de page.

II9. S. Reinach, Catalogue illustré du Musée des Antiquités Nationales au château de Saint-Germain-en-Laye, p. 304. Sur le trésor de la collection Messaksoudy, voir encore 


\subsubsection{Présentation de la collection au public}

Lors de son acquisition, la collection Messaksoudy a été somptueusement présentée. Au Louvre, la collection est installée vers le début du mois de juillet 1920, dans sa totalité. Lors de la séance de la Société Nationale des Antiquaires de France du 7 juillet 1920, Étienne Michon fait une communication aux membres de cette institution:

Il y a là, vous l'avez peut-être admiré, tout un ensemble d'antiquités de la plus haute importance recueillies dans la région de Kertch, ensemble dont la valeur scientifique et documentaire se double par l'effet même de leur réunion et dont, en dehors des musées russes, on ne trouverait nulle part l'équivalent ${ }^{120}$.

La collection reste exposée en l'état probablement jusqu'en janvier 1921, lorsque $S$. Reinach demande de récupérer le «tombeau gothique» provenant de la collection Messaksoudy pour le musée de Saint-Germain, qui sera présenté dans la salle Numismatique (dite également "Du Trésor» ou «Des Bijoux») ${ }^{121}$. La presse se fait l'écho de cette acquisition et de sa présentation. La collection Messaksoudy fait aussi l'objet d'une vraie campagne photographique, comme on peut le constater dans la documentation du DAGER: de nombreuses photos montrent les objets exposés dans des vitrines aménagées spécialement au milieu de la Salle des Sept-Cheminées. Pareil effet muséographique doit donner toute la mesure de l'attention et du prestige conféré à cet achat: la collection Messaksoudy est entourée de tableaux prestigieux, comme le Sacre de l'empereur Napoléon I Ier par David d'un côté, Napoléon sur le champ de bataille d'Eylau par Gros de l'autre.

Le lundi 25 janvier 1921, le Louvre inaugure la Salle des Bijoux Antiques qui regroupe, autour de la collection Campana, les acquisitions postérieures, entre autres "celles qui ont été acquises récemment du collectionneur russe Messaksoudis». La collection y est donc temporairement transférée de la Salle des Sept-Cheminées, toujours mise en

M. Rostovtzeff, «Quelques objets de la collection Messaksoudi entré au Louvre et au musée de Saint-Germain » et «Une trouvaille de l'époque gréco-sarmate de Kertch au Louvre et au musée de Saint-Germain».

I20. Bulletin de la Société Nationale des Antiquaires de France, Extrait des ProcèsVerbaux du III trimestre de 1920, séance du 7 juillet, p. 257.

I2I. S. Reinach, Catalogue illustré du Musée des Antiquités Nationales au château de Saint-Germain-en-Laye, p. 304. 
exergue et entourée d'œuvres parmi les plus prestigieuses du Louvre ${ }^{122}$. À Saint-Germain-en-Laye, la collection Messaksoudy est installée dans la Salle Numismatique, en vitrine 5 , soit à un emplacement prestigieux à l'époque ${ }^{123}$. Salomon Reinach rédige le catalogue du musée et décrit en détail les objets ayant constitué le mobilier de la tombe princière: «l'épée d'apparat à poignée d'argent et garde en jade», la pyxide de cuivre émaillée, la ceinture de cuir, la boucle de ceinture et la couronne en or - autant de pièces qu'il réclamait dès le début pour le musée (fig. 7). Suite à l'achat de la totalité de la collection, le "Musée en a reçu d'autres de la même collection: grande boucle de ceinturon en argent, broche en argent plaqué, deux grandes boucles d'oreilles d'or fourré, ornées de verres de couleurs etc. » ${ }^{124}$. Ajoutons ici que les pièces les plus prestigieuses de la collection ont fait l'objet d'une publication dans le luxueux ouvrage Les accroissements des Musées Nationaux pour l'année 1920: Les divinités d'Éleusis sur un lécythe à reliefs de Crimée, La naissance de Vénus sur une onochoé à reliefs, L'héroïne Atalante sur une coupe de style attique, La pyxis émaillée de Kertch ${ }^{125}$.

\section{Conclusion}

Miroir de l'intérêt porté par l'archéologie classique française et des rapports qu'elle entretient avec cette partie excentrée du monde grec, tout

I22. Le Temps du 23 janvier 1921, p. 4, rubrique Art et Curiosités, article Au musée du Louvre. De nos jours, il n'est plus possible d'admirer cette collection dans sa totalité. Ses pièces sont en effet dispersées dans différentes salles du département. Il faut, pour la parcourir, se déplacer dans l'aile Sully, entre la Galerie Campana au premier étage, la salle Clarac (salle 35, vitrine 4: Bois, stucs et verres de Kertch [ancienne Panticapée]), la salle 38 dans les actuelles salles Charles X du département des AGER ouvertes en 1997 (vitrine 14: La comédie antique) ou dans la Salle de verres, ainsi que dans la Salle des Bronzes (vitrine C5: Bijoux qui comprend également une entité dénommée Bosphore Cimmérien et Orient hellénisé).

I23. S. Reinach, Catalogue illustré du Musée des Antiquités Nationales au château de Saint-Germain-en-Laye, p. 304 sqq. Aujourd'hui la salle est inaccessible aux visiteurs, elle se trouve dans la partie destinée aux réserves. Je tiens à remercier la conservatrice, Madame Anaïs Boucher, pour son aimable accueil.

I24. Aujourd'hui, certains objets de la collection sont exposés dans la Salle d'Archéologie comparée, dans la vitrine Naissance de l'Europe, mais aucune indication n'est faite sur leur provenance, conformément à la pratique qui prévaut pour les cartels du musée.

I25. H. Rivière, Les accroissements des musées nationaux français, t. $3: \mathrm{pl}^{\circ} \mathrm{n}^{\circ} 33$ et 34 (CA 2271 du Louvre); pl. no 32 (CA 2259 du Louvre); pl. nº 35 (nº inv. : 66.112 au M.A.N.). 
Fig. 7 - M. Rostovtzeff, "Une trouvaille de l'époque gréco-sarmate de Kertch au Louvre et au musée de Saint-Germain", in Fondation Eugène Piot, Monuments et mémoires, Paris, Éditions Ernest Leroux, 1923, t. 26, pl. IV. 
comme de la concurrence tenace entre les institutions muséales européennes, et notamment allemandes, les Antiquités provenant de la mer Noire ont été reléguées pendant longtemps au second plan, car elles ne bénéficiaient pas du prestige esthétique grec tel qu'il était conçu à l'époque. Leur introduction dans le champ de l'étude des collections françaises s'avère à ce titre relativement récente.

Force est de constater que les acquisitions n'ont pas été progressives, mais irrégulières et qu'elles se sont articulées autour de deux temps forts: la campagne de Crimée et la vente de la collection Messaksoudy, qui toutes deux représentent plus de $85 \%$ du fonds du musée relatif à cette région. Alors que ces objets sont arrivés au Louvre à la suite des soubresauts de l'histoire européenne et furent accueillis parfois sans grande conviction, on observe que seule leur valeur esthétique a longtemps été prise en compte: ce fut le cas lors de l'achat de la collection Messaksoudy, où seules les pièces maîtresses ont d'abord intéressé les conservateurs avant l'acquisition complète de la collection.

Pour des raisons historiques, les savants allemands investirent assez rapidement le terrain de l'archéologie pontique et cette situation se traduisit par l'acquisition active d'antiquités venant de cette région - une attitude qui ne fut adoptée que plus tard par les milieux savants français. Cependant, la curiosité de quelques esprits, aiguisée sans doute par la parution de l'ouvrage de Salomon Reinach sur les Antiquités du Bosphore Cimmérien ${ }^{126}$, alliée à une plus large connaissance des collections criméennes de l'Ermitage, diffusée par la Gazette des Beaux-arts, contribuèrent à l'intérêt des conservateurs du Louvre à cette région du monde gréco-romain et participèrent à sa documentation au sein du musée.

Au regard des critères appliqués à l'étude des collections provenant d'Italie ou de Grèce, ces antiques, considérés dans leur globalité, présentaient pourtant des similitudes manifestes et pouvaient légitimement prétendre à leur pleine insertion dans le champ des études muséologiques françaises et plus largement occidentales.

Joanna MarTin

Université de Paris-Nanterre et École du Louvre

I26. S. Reinach, Antiquités du Bosphore Cimmérien. 


\section{BIBLIOGRAPHIE}

Andrasi, Julia, Aibabin, Aleksander, The Berthier-Delagarde Collection of Crimean Jewllery in the British Museum and Related Material, London, British Museum, 2008.

Antiquités du Bosphore Cimmérien conservées au Musée Impérial de l'Ermitage. Ouvrage publié par ordre de sa Majesté l'Empereur, Saint-Pétersbourg, imprimerie de l'Académie Impériale des Sciences, 1854.

Arveiller-Dulong, Véronique, Nenna, Marie-Dominique, Les verres antiques du musée du Louvre. II: Vaisselle et contenants du I Ier siècle au début du VII siècle après J.-C., Paris, Somogy/Musée du Louvre, 2006.

Bodzek, Jarosław, "Coins from Greek Cities on the Northern Coast of the Black Sea in the Collection of the National Museum in Cracow", Studies in Ancient Art and Civilization, 8 (1997), p. 61-69.

Borovkova, Viktorija Nikolaevna, Kollekcionery i torgovcy kerčenskimi drevnostjami, Kerch, Demetra, 1999.

Bresc, Geneviève, Pingeot, Anne, Sculptures des jardins du Louvre, du Carrousel et des Tuileries, Paris, Éditions de la Réunion des Musées nationaux, 1986, (Notes et documents des Musées de France, 12).

Byкоvsкaja, Natalja Vladimirovna, "Grečeskaja obščina v èkonomičeskom razvitii Kerč-Enikal'skogo gradonačal'stva $\mathrm{v}$ konce XVIII - načale XX veka» ["La communauté grecque et le développement économique du territoire de Kertch-Yenikale à la fin du XVIII - début du XX ${ }^{\mathrm{e}}$ siècle»], in Grec'ke pidpryêmnyctvo i torgivlja u Pivničnomu Pryčornomor'ï XVIII - XIX st. [L'entrepreneuriat grec et le commerce en mer Noire septentrionale du XVIII ${ }^{e}$ au XIX ${ }^{e}$ siècle], otv. red. Gennadij Volodymyrovyč Borjak, Kyïv, Instytut istoriï Ukraïny, 2012, p. 49-60.

Byкоvsкaja, Natalja Vladimirovna, Sanžarovec, Vladimir Filipovič, "Kerčenskij tabačnij fabrikant Konstantin Mesaksudi» [«Konstantin Mesaksudi, un fabricant de tabac de Kertch»], in 
Podvižnyky j mecenaty. Grec'ki pidpryêmci ta gromads'ki dijači v Ukraïni XVII-XIX st. [Pionniers et mécènes. Entrepreneurs grecs et personnalités publiques en Ukraine du XVII e au XIX e siècle], otv. red. Valerij Andrijovyč Smolij, Kyïv, Instytut istoriï Ukraïny, 2001, p. 176-189.

Callu, Agnès, La Réunion des Musées nationaux, Paris, École des Chartes, 1994, (Mémoires et documents de l'École des Chartes, 42).

Fedoseev, Nikolaj Fjodorovič, Kerčenskij muzej drevnostej [Le musée des antiquités de Kertch], en ligne: <http://www.kerch-museum.com/ userfiles/VDI.pdf> [consulté le 14 avril 2014].

Frel, Jiři, "Miscellanea Pontica I», Sbornik praci filozofické fakulty brnènské univerzity, E 6, t. 10 (1961), p. [155]-168.

—, «Miscellanea Pontica II», Sborník prací filozofické fakulty brnènské univerzity, E 8, t. 12 (1963), p. [93]-96.

Fumaroli, Marc, «Retour à l'Antique: la guerre des goûts dans l'Europe des Lumières", in L'Antiquité rêvée. Innovations et résistances au XVIIIe siècle, éd. par Guillaume Faroult, Christophe Leribault, Guilhem Scherf, catalogue d'exposition, Paris, Gallimard/Les éditions du musée du Louvre, 2010, p. 23-55.

Guérin, Léon, Histoire de la dernière guerre de Russie (1853-1856), Paris, Dufour, Mulat et Boulanger Éditeurs, 1858.

Kazanski, Michel, Beck, Françoise, Vallet, Françoise, «La riche tombe de Kertch du Musée des Antiquités Nationales", Antiquités Nationales, 20 (1989), p. 63-81.

La Bédollière, Émile de, Histoire de la guerre d'Orient, Ge Série, Malakoff, Paris, G. Barba, (s. d.), (Le Panthéon populaire illustré). Lenormant, Charles, "Mémoire sur les antiquités du Bosphore Cimmérien, à propos de l'ouvrage publié par le gouvernement russe", Comptes rendus des séances de l'Académie des Inscriptions et Belles-Lettres, 3 (1859), p. 52-66.

Leskov, Aleksander, The Maïkop Treasure, Philadelphia, University of Pennsylvania Museum of Archaeology and Anthroplogy, 2008.

Lintz, Yannick, "Les apports du récolement à la connaissance des collections", Journées d'étude sur le récolement décennal dans les musées nationaux, Saint-Germain-en-Laye, 24-25 novembre 2011, en ligne: <http://www.culture.gouv.fr/documentation/joconde/ 
fr/partenaires/AIDEMUSEES/journee_RD_2011/lintz.htm> [consulté le 30 mars 2014].

Manuel général de l'instruction primaire, 24 (16 juin 1855), en ligne $<$ http://numerisation.bibliotheque-diderot.fr/R/5KM989E MGT1R2ATVVDPX7BX77XPKAPEIS46D13X1IAPJV G1P1-01693?func=results-jump-full\&set_entry=000024\&set_ number $=000027 \&$ base $=$ GEN01-DID01 $>$.

McPherson, Duncan, Antiquities of Kertch and researches in the Cimmerian Bosphorus, London, Smith, Elder and Co., 1857.

Orgogozo, Christine, Lintz, Yannick (dir.), Vases, bronzes, marbres et autres antiques. Dépôts du musée du Louvre en 1875: étude historique et catalogue, Paris, Gourcouff Gradenigo/Musée du Louvre, 2007.

PASQUier, Alain, "Une nouvelle acquisition du musée du Louvre: une tête de cheval archaïque en marbre (note d'information)", Comptes rendus des séances de l'Académie des Inscriptions et BellesLettres, 149-1 (2005), p. 159-172.

Perret, Eugène, Les Français en Orient. Récits de Crimée, 1854-1856, Paris, Bloud et Barral, [1888].

PICK, Eugène (de l'Isère), Les fastes de la guerre d'Orient. Histoire politique, militaire et maritime des campagnes de Crimée [...], Paris, Librairie napoléonienne des arts et de l'industrie, 1856.

Pinelli, Paule, WĄsOWICZ, Aleksandra, Catalogue des bois et stucs grecs et romains provenant de Kertch, Paris, Éditions de la Réunion des musées nationaux, 1986.

Rapport de M. le Comte de Nieuwerkerke, surintendant des Beaux-Arts, membre de l'Institut sur les travaux de remaniement et d'accroissement réalisés depuis 1849 dans les Musées Impériaux suivi d'un relevé sommaire des objets d'art entrés dans les collections de 1849 à 1863, Paris, Librairie académique/Didier et Cie, libraireséditeurs, 1863.

Reinach, Salomon, Antiquités du Bosphore Cimmérien (1854), rééditées avec un commentaire nouveau et un index général des comptes rendus, Paris, Librairie de Firmin-Didot et C $\mathrm{C}^{\mathrm{ie}}, 1892$.

-, Catalogue illustré du Musée des Antiquités Nationales au château de Saint-Germain-en-Laye, t. 2, Paris, Musées Nationaux, 1921.

Ridder, André de, Catalogue sommaire des bijoux antiques, Paris, Musées nationaux, 1924. 
Rivière, Henri, Les accroissements des musées nationaux français: le Musée du Louvre, Paris, Demotte, 1919-1921.

Rostovtzeff, Mikhail, «Quelques objets de la collection Messaksoudi entré au Louvre et au musée de Saint-Germain", Comptes rendus des séances de l'Académie des Inscriptions et Belles-Lettres, 66-1 (1922), p. 61.

—, «Une trouvaille de l'époque gréco-sarmate de Kertch au Louvre et au musée de Saint-Germain", in Fondation Eugène Piot, Monuments et mémoires, Paris, Éditions Ernest Leroux, 1923, t. 26, p. 100-163.

Schiltz, Véronique, "Du bonnet d'Ulysse à la tiare de Saïtapharnès", in The traditions of East and West in the antique cultures of Central Asia. Papers in Honor of Paul Bernard, ed. by Kazim Abdullaev, Tachkent, Noshirlik yog'dusi' Press, 2010, p. 217-234.

Szemiothowa, Anna, Numizmatyka starożytna. Katalog wystawy statej [Numismatique antique. Catalogue de l'exposition permanente], Warszawa, Wydawnictwo Muzeum Narodowe w Warszawie, 1951.

—, «Historia powstania działu numizmatyki starożytnej Muzeum narodowego w Warszawie» ["Histoire de la création du département de numismatique antique au Musée national de Varsovie»], Rocznik Muzeum narodowego w Warszawie, 3 (1958), p. 335-351.

Tunkina, Irina Vladimirovna, Russkaja nauka o klassičeskikh drevnostjakh juga Rossii (XVIII - seredina XIX vv.) [La recherche scientifique russe sur les antiquités classiques de la Russie méridionale (XVIII première moitié du XIX ${ }^{e}$ siècle)], Sankt-Peterburg, Nauka, 2002. 